\title{
Das Nationalismusprinzip in der türkischen Verfassung
}

\author{
Von Christian Rumpf 1
}

\section{Einleitung: Hintergrund der Fragestellung}

Als zentralistischer Einheitsstaat hat die Republik Türkei über Jahrzehnte den Eindruck zu erwecken versucht, eine Nationalitätenfrage gebe es auf ihrem Boden nicht. Vor allem die Leugnung der Existenz mehrerer Millionen Kurden - noch zu Beginn der Republik eine anerkannte Selbstverständlichkeit - trieb ihre seltsamen Blüten, die der Idee des Nationalismus, unter dem Republikgründer Mustafa Kemal Atatürk zum staatstragenden Grundsatz erhoben, ein schillerndes Gepräge gaben und letztlich dem Verdacht aussetzten, nicht nur ein ideologischer Grundsatz zum Zweck der Modemisierung einer gegenüber Westeuropa zurückgebliebenen Gesellschaft, sondern auch ein Vehikel zur zwangsweisen Assimilation kulturell unterschiedlicher Völkerschaften zu sein.

In den letzten drei Jahren hat sich jedoch in der Türkei bezüglich der Diskussion der Kurdenfrage ein deutlicher Wandel vollzogen. Versuche, die Prozesse der öffentlichen Meinungsbildung durch eine spezifische Notstandsrechtspolitik zu kanalisieren und gegebenenfalls zu unterdrücken ${ }^{2}$, sind nicht nur ohne spürbares Ergebnis geblieben, sondem haben das Gegenteil erreicht. Seit ungefähr Mitte 1990 verlor die türkische Presse zunehmend die Scheu, das soziale und wirtschaftliche Problem auch mit ethnischen Faktoren in Zusammenhang zu bringen und die Öfentlichkeit mehr denn je mit der "kurdischen Realität" zu konfrontieren. Den endgültigen Durchbruch konnte man im Frühjahr 1991 verzeichnen, als die Türkei mehrere Hunderttausend irakischer Kurden als Flüchtlinge aufnahm und damit

1 Abkürzungen: AMKD (Anayasa Mahkemesi Kararlarì Dergisi - Entscheidungssammlung des Verfassungsgerichts); ATG (Antiterrorgesetz); E. (Esas - Rechtssache); EuGRZ (Europäische Grundrechte Zeitschrift); InfAuslR (Informationsbrief Ausländerrecht); IÜHFM (Istanbul Üniversitesi Hukuk Fakültesi Mecmuasì - Zeitschrift der Juristischen Fakultät der Universität Istanbul [auch IÜHFD]); JöR (Jahrbuch des öffentlichen Rechts - Neue Folge); K. (Karar - Entscheidung); MÇP (Milliyetçi Çalìsma Partisi - Nationalistische Partei der Arbeit); RG (Resmî Gazete - türk. Amtsblatt); TBKP (Türkiye Birlesik Komünist Partisi - Vereinigte Kommunistische Partei der Türkei); TV (türkische Verfassung); Urt. (Urteil); VerfG (Verfassungsgericht); VRÜ (Verfassung und Recht in Übersee); ZaöRV (Zeitschrift für ausländisches öffentliches Recht und Völkerrecht); ZAR (Zeitschrift für Ausländerrecht und Ausländerpolitik); ZfP (Zeitschrift für Politik); ZfTS (Zeitschrift für Türkeistudien)

2 Vgl. Christian Rumpf, Notstandsdiktatur in Teilen der Türkei und ihre rechtlichen Auswirkungen auf das Regime der Normalverfassung im übrigen Staatsgebiet der Republik, EuGRZ 1990, S. 249 ff. 
der nationalen Öfentlichkeit vor Augen führte, was der internationalen Öfentlichkeit schon lange bewußt war: Die irakischen Flüchtlinge - die als "Kurden" zu bezeichnen sich auch die "offizielle" Türkei nie gescheut hatte - sprechen, wenn auch in Varianten, die gleiche Sprache wie die örtliche türkische Bevölkerung.

Etwa zu gleicher Zeit, nämlich am 12. April 1991, wurde ein Gesetz zur Bekämpfung des Terrors im Amtsblatt verkündet ${ }^{3}$, das zu einer gewissen Reform des politischen Strafrechts führte und zur weiteren Öffnung der Disskussion um die Kurdenfrage beitrug, obwohl als Relikt des alten Gesinnungsstrafrechts "separatistische Propaganda" nach wie vor strafrechtlich sanktioniert blieb. Schließlich kam es im Herbst 1991 zu einem Regierungswechsel zugunsten einer konservativ-sozialdemokratischen Koalition, die die friedliche Lösung der Kurdenfrage in ihr Programm aufnahm.

Damit entstand der Eindruck, daß nun doch eine friedliche Lösung der Kurdenfrage unter Berücksichtigung eines Maximums an kultureller Eigenständigkeit im Rahmen des unitarischen Zentralstaates kemalistischer Prägung möglich sei. Die türkische Verfassungsordnung scheint jedoch auf solche Entwicklungen nicht vorbereitet zu sein.

\section{Der Kemalismus}

\section{Vorbemerkung}

Der Kemalismus ist eine dynamische Staatsauffassung, die in der Lage ist, sich nach den Regeln politischer Vemunft den Erfordemissen der Gegenwart anzupassen. Sie ist damit geeignet, den schweren Schaden, den nationalistisches Gedankengut zulasten eines der berühmtesten Wahlsprüche Mustafa Kemal Atatürks, "Frieden im Lande und Frieden in der Welt", anrichtet, zu vermeiden. Kompromißlos ist diese Staatsauffassung nur insoweit, als sie eine Abkehr von abendländischen Idealen und Hinwendung zur osmanischen Tradition nicht zuläßt. Mit anderen Ideologien teilt aber der Kemalismus auch den Mangel, daß er oft als Legitimationsgrundlage für politische Maßnahmen herzuhalten hat, die lediglich den Interessen einer gegenwärtigen politischen oder Staatselite bzw. nur eines kleinen herrschenden Teils derselben und damit zu nichts anderem als der Erfüllung der Interessen kleiner Gruppen oder einzelner Personen in höchsten Ämtern dienen, nicht aber dem Wohl der Gesamtbevölkerung und der Erhaltung humanistischer Grundwerte.

3 Dazu Bülent Tanör, Gedanken zum türkischen Gesetz Nr. 3713 zur Bekämpfung des Terrors ("Antiterrorgesetz" - ATG), ZfTS 1991, S. 153 ff.; Sylvia Tellenbach, Das türkische Antiterrorgesetz, ZAR 1991, S. 162 ff.; Christian Rumpf, Das türkische Gesetz zur Bekämpfung des Terrors (Antiterrorgesetz), Inf AuslR 1991, S. 285 ff. 
Solange der Kemalismus nicht als Ideologie zugunsten privater Interessen instrumentalisiert und als Mittel autoritärer Herrschaftsausübung mißbraucht wird, scheint der Kemalismus grundsätzlich durchaus geeignet, rationaler türkischer Politik Impulse zu geben 4 . Dies setzt voraus, daß ihm die einer Ideologie eigene sakrale Uberhöhung genommen und die Eigenschaft einer modernen, dem Demokratieprinzip verpflichteten Weltanschauung zugeschrieben wird5. Statt dessen birgt gegenwärtig die türkische Verfassung die durchaus konkrete Gefahr in sich, als Verfassung eines ideologisierten und damit besonders starren politischen Systems den "Kemalismus" zur Antipode gegenüber freiheitsorientierter, demokratischer Herrschaftsausübung zu machen, ihn gleichzusetzen mit einem unangemessenen Autoritarismus, der ihn schnell als Ideologie in die Nähe des spanischen oder italienischen Faschismus rückt und damit diskreditiert. Die Auflösung dieses normativen Konflikts ist eine der vordringlichen Aufgaben künftiger türkischer Verfassungspolitik. Das Hauptproblem dabei ist der Umgang mit dem "Nationalismus" als Teilgrundsatz des Kemalismus und in seiner Ausprägung in Text und Wirklichkeit der türkischen Verfassung, der voller Widersprüche steckt, die noch aufzulösen sind.

\section{Die Grundzüge des Kemalismus}

Um "den" Kemalismus wirklich zu begreifen, wäre es notwendig, die gesamte Zeit, in der Mustafa Kemal Atatürk als Vater dieser Ideologie gewirkt hat, zu untersuchen, nämlich die Zeit vom Beginn des Unabhängigkeitskampfes am 19.5.1919 (Landung in Samsun) bis zu Atatürks Tod am 10.11.19386. Ja man müßte noch weiter ausholen, um zu erfassen, inwieweit der Kemalismus mit den Modemisierungsbestrebungen der Jungtürken in Zusammenhang steht 7 . Eine solche Untersuchung kann im Rahmen eines begrenzten Beitrags wie diesem nicht geleistet werden. Denn im Laufe dieser zwanzig Jahre hat der Kemalismus

4 Zu diesem Ergebnis kommt auch Baskin Oran in seiner kritischen Studie: Atatürk Milliyetçiligi Resmî Ideoloji Dìsì Bir Inceleme (Der Nationalismus Atatürks - Eine Untersuchung außerhalb der offziellen Ideologie), 1. Aufl., Ankara 1988, S. 241.

5 Metin Heper, A Weltanschauung-tumed Partial Ideology and Normative Ethics: "Atatürkism" in Turkey, in: Orient 1984, S. 83 ff. In der Tat war die "Weltanschauung" Mustafa Kemal Pascha zu eigen; seine Diadochen sorgten für die Ideologisierung. Allgemein hierzu auch Esat Çam, Ideoloji olarak Kemalizm (Der Kemalismus als Ideologie), Gedenkschrift für H. Furgaç, Istanbul 1974, S. 159 ff. Auch die übrige türkische Literatur (siehe die weiteren Fn.) geht vom Kemalismus als Ideologie aus. Als "pragmatisch-demokratische" Modemisierungsideologie wird der Kemalismus von Ismet Giritli, Kemalizm: "Pragmatik-Demokratik" Modemlesme Ideolojisi, Tageszeitung Milliyet v. 3.6.1981, S. 2 bezeichnet.

6 Vgl. den Sammelband Mustafa Kemal Atatürk (1881-1938), Vorträge und Aufsätze zu seinem 100. Geburtstag, Heidelberg 1982.

7 Vgl. Levent Köker, Modemlesme, Kemalizm ve Demokrasi (Modemisierung, Kemalismus und Demokratie), Istanbul 1990, S. 67 ff., der die Zusammenhänge noch weiter zurückverfolgt. 
Facetten entwickelt, die nicht nur zahlreich, sondern auch widersprüchlich sind ${ }^{8}$. Man mag es so zusammenfassen: Die Lehren des Kemalismus zeichneten sich durch ihren Pragmatismus aus und richteten sich nach den aktuellen politischen Erfordemissen. Zu Beginn gab es das "Kurdenproblem" nicht, weil man davon ausging, daß die Kurden "mit der türkischen Regierung einverstanden" seien. Mit dieser Auffassung ist bei den Verhandlungen zurn Lausanner Vertrag die damalige türkische Delegation auch bei den westlichen Verhandlungspartnem durchgedrungen 9 . Entscheidend ist hier, daß die Kurden als eigene Ethnie anerkannt waren. Die Leugnung der Kurden als ethnische Minderheit begann erst sehr viel später, selbst die Siedlungsgesetzgebung der Jahre 1934 und 1935 ging ganz offenbar noch von zumindest kulturell verschiedenen Volksgruppen aus. Auch im Verhältnis zum Islam zeigte sich der Kemalismus mit verschiedenen Varianten der laizistischen Idee und ihrer Durchsetzung flexibel. Es kam insgesamt allein darauf an, der neuen Türkei auf ihrem im Unabhängigkeitskrieg erhalten gebliebenen Staatsgebiet das Wesen eines modernen Staates zu vermitteln, das diesem Staat eine angesehene und gleichwertige Stellung im Konzert der Weltgemeinschaft der souveränen Staaten sichern konnte10. Und diese Völkergemeinschaft war in den zwanziger und dreißiger Jahren weitgehend identisch mit der Gemeinschaft der europäischen Staaten. Denn Europa war es, das bis vor kurzem die Regeln des Völkerrechts bestimmt hatte. Selbst die Vereinigten Staaten von Amerika waren erst mit dem Ersten Weltkrieg erfolgreich in diese Gemeinschaft hineingestoßen. Es fehlte praktisch noch die gesamte Dritte Welt, die Rolle Japans und Chinas war begrenzt, Südamerika blieb - unter dem Einfluß der Vereinigten Staaten - weitgehend unter sich. Die als "Kemalismus" bezeichnete Staatsauffassung des Begründers der modernen Türkei, Mustafa Kemal Atatürk, ist eine Idee, die den Versuch des Bruchs mit den geschichtlichen Traditionen des Osmanischen Reiches voraussetzt und auf eine Synthese zwischen der Verinnerlichung der Werte und Ordnungen des europäisch-abendländischen Kulturkreises und dem erst um die letzte Jahrhundertwende wieder neu entdeckten Türkentum ausgerichtet ist.

Das Ideal einer solchen Synthese ist allerdings bis heute nicht erreicht worden. Dazu war die Zeit bisher zu kurz und der Kemalismus in seiner Durchdringung des gesamten sozialen

8 Vgl. aus türkischer Sicht: Tekin Alp, Le Kémalisme, Paris 1937; Papers and Discussion - Türkiye Is Bankasì. Intemational Symposium on Atatürk, Ankara 1984; Ibrahim Kafesoglu/Mehmet Saray, (Hrsg.), Atatürk Ilkeleri ve Dayandigì Tarihî Temeller (Die Prinzipien Atatürks und ihre historischen Grundlagen), Istanbul 1983; Asim Aslan, Sömürülen Atatürk ve Atatürkçülük (Die Ausbeutung von Atatürk und Kemalismus), 5. Aufl., Ankara 1989; Abdurrahman Dilipak, Bir Baska Açìdan Kemalizm (Der Kemalismus aus anderer Sicht), Istanbul 3. Aufl. 1988; Ismet Giritli, Atatürkçülük (Der Kemalismus), Istanbul 1987.

9 Vgl. etwa die Verhandlungen zum Lausanner Vertrag, wiedergegeben bei Seha L. Meray, Lozan Baris Konferansì. Tutanaklar ve Belgeler (Die Friedenskonferenz von Lausanne, Verhandlungsprotokolle und Dokumente), Serie I, Bd. 1, Buch 1 und 2, Ankara 1973, hier: Buch 1, S. 301 f. und Buch 2, S. 154 (Kurden und Türken als Schicksalsgemeinschaft).

10 Vgl. etwa auch die Präambeln zu den Verfassungen von 1961 und 1982. 
Lebens nicht radikal genug ${ }^{11}$. Es hat sich gezeigt, daß sich das "Türkentum"-Verständnis von religiös bestimmten kulturellen Elementen nicht völlig freihalten läßt, wodurch gewisse Verknüpfungen mit dem Islam bestehen bleiben, die sogar bis in die Verfassung hineinreichen, wie nachher nachgewiesen werden wird. Die religiösen, aus der orientalischarabischen Tradition überlieferten Werte des Islam können daher nach wie vor weite Teile des sozialen Lebens der Mehrheit der türkischen Bevölkerung bestimmen und damit eine Verwirklichung abendländischer Werte verhindern, falls der Islam selbst nicht - ähnlich den Kirchen im Zeitalter der Reformation und Aufklärung - Kompromisse und neue Geistesströmungen zuläßt.

Der Kemalismus fand zum ersten Mal mit seinen wesentlichen Grundsätzen - im damaligen Parteiprogramm der Republikanischen Volkspartei und ihrem Emblem symbolisiert durch die "sechs Pfeile" - im Jahre 1937 Eingang in die Verfassung von 1924. Diese Grundsätze waren der Wegweiser für das vorrangige und wesentliche Ziel für den neuen türkischen Staat, das auch heute noch nicht als völlig erreicht angesehen wird: die Schaffung eines türkischen Nationalstaats, der in der Lage ist, eine politisch und wirtschaftlich selbständige Position unter den Staaten der Welt einzunehmen und den eigenen Bürgern ein friedliches und menschenwürdiges Auskommen sowie den Standard moderner Zivilisation zu vermitteln.

Diese Grundsätze sind: der Republikanismus als Absage an das alte monarchische System der Herrschaft eines souveränen Herrscherhauses (vgl. schon Art. 1 TV 1924) zugunsten eines auf der Volkssouveränität beruhenden Regimes; der Nationalismus als ideelles Band, welches das Staatsvolk der Republik Türkei zu einer Einheit verschmelzen und es als Nation zum Träger der staatsrechtlichen Souveränität und des völkerrechtlichen Selbstbestimmungsrechts werden lassen soll; der Populismus (Volksverbundenheit) als Ausdruck der Idee einer Herrschaft durch und mit dem Volk statt über das Volk; der Etatismus - gelegentlich auch als Interventionismus bezeichnet - als Prinzip, das dem Staat das Recht und die Pflicht zuweist, zum Zweck des Aufbaus der Wirtschaft als materieller Grundlage des modernen Staates dirigistische Maßnahmen zu ergreifen; der Laizismus als Prinzip der Trennung von Religion und Staat; der revolutionäre Reformismus als dynamisches Prinzip, das der Verwirklichung der übrigen Grundsätze dient und auf die Anpassung der politischen, gesellschaftlichen, wirtschaftlichen und rechtlichen Verhältnisse an die Erfordernisse eines modernen europäischen Staates abzielt.

Was diese "sechs Pfeile" ausdrücken, sind Funktionen. Dabei wird der eher statisch anmutende Charakter der ersten fünf Elemente durch den dynamistischen Kern des letzten Ele-

11 Vgl. auch E. Özbudun, Social Change and Political Participation in Turkey, Princeton 1976, S. 43. 
ments, des revolutionären Reformismus, in Fluß gebracht ${ }^{12}$. Als Funktionen dienen sie einem freiheits- und gemeinwohlorientierten "Zivilisationismus" - dieser Ausdruck hat in dem monströsen türkischen Wort "medeniyetçilik" seinen Niederschlag in der Präambel der türkischen Verfassung von 1982 gefunden -, der eine flexible Anpassung des türkischen Staates und seines Staatsvolkes an die gegebenen politischen, sozialen, wirtschaftlichen und kulturellen Standards und Errungenschaften der modernen westlichen Gesellschaft ermöglichen soll. Nicht zu vergessen ist dabei auch die Anspielung auf die Verbindung der abendländischen Kultur mit den alten kleinasiatischen Zivilisationen. Das dynamische Moment ist insbesondere dort hervorzuheben, wo das eine oder andere Element des Kemalismus von den politisch herrschenden Kräften "konservativ" aufgefaßt und in diesem Sinne seine Umsetzung versucht wird: zum Zweck der Erhaltung eines status quo, der sich unversehens von jenen Standards und Strömungen modemen Denkens, wie sie sich in den europäischen Staaten in den Bereichen von Politik, Wirtschaft, Recht und Kultur durchsetzen, entfernt. So kann ein strikter Laizismus kontraproduktiv wirken, wenn religiöse Kräfte unterdrückt statt absorbiert werden und sie so latente Sprengkraft entfalten. Gleiches gilt für einen Etatismus, der in einer ungleichgewichtigen Wirtschaft mit geringer Produktivität und fehlender Ausschöpfung oder verbreitetem Mißbrauch vorhandener Potentiale und Reserven seine Berechtigung haben mag, aber in einer zwar standardisierten, aber nicht "gegängelten" sozialen Marktwirtschaft die Entwicklung neuer Produktivkräfte und die Reaktion auf neue, für den internationalen (und nationalen) Wirtschaftsverkehr bedeutsame Umstände neu zu definieren ist. Das gilt schließlich für den Nationalismus, der je nach zeitlichen und sachlichen Umständen verschiedenen Herausforderungen ausgesetzt ist, je nachdem, ob er der Herbeiführung einer noch fehlenden Integration eines Staatsvolkes dient, oder nach weitgehend gelungener Integration sich auf die Abgrenzung nach außen konzentriert.

\section{Der Kemalismus als Ideologie}

"Eine Gesellschaft kann nur existieren, wenn sie sich bestimmte Vorstellungen von sich selbst macht und sich in dieser Selbstinterpretation einen Wert zuschreibt. Diese Vorstellung nennt man gewöhnlich Ideologie"13. Die klassischen Ideologien gehen aber gewöhnlich darüber hinaus. Sie schreiben sich einen bestimmten gesellschaftlichen Zustand als Bedingungsrahmen und die Funktion zu, diese Bedingungen im Sinne einer Idee oder eines Ideals zu gestalten, das auf einer Zukunftsprognose beruhen soll, die wissenschaftlich fundiert und an einer aufgeklärten Ethik der Freiheit und Gleichheit (Marxismus) oder

12 Vgl. dazu etwa Suna Kili, Atatürk Ilkeleri (Die Prinzipien Atatürks), 2. Aufl. Ankara 1981, S. $274 \mathrm{ff}$.

13 Jean Lacroix, zit. bei Alfred Grosser, Politik erklären, München 1973, S. 57. 
emotional begründet und an einem bestimmten "schönen" Menschenbild orientiert ist (Turanismus; Rassismus der Nationalsozialisten).

Oder aber es wird eine bestimmte Staatsraison aus konkretem politisch-historischem Anlaß und im Rahmen bestimmter gesellschaftlicher und wirtschaftlicher Bedingungen zur Ideologie aufgewertet, um ihr die Funktion zu vermitteln, die Gestaltung bzw. Veränderung der gegebenen Bedingungen an konkreten, durch lebende Vorbilder gespeisten Vorstellungen zu orientieren und voranzutreiben 14. Gerade im Hinblick auf den Kemalismus könnte man hier auch von einer pragmatisch fundierten und orientierten Ideologie oder von einer Entwicklungsideologie sprechen, deren Funktion die Modemisierung ist 15.

Ideologie wirkt trotz der entgegengesetzten, sich selbst zugeschriebenen Funktion der Veränderung antidynamisch, indem sie versucht und dazu dient, den Menschen in seinem seiner Natur nach unruhigen Geist einzufangen und ihm damit seine produktive Beweglichkeit zu nehmen - desto mehr, umso weiter das postulierte System ausgestaltet ist und den Menschen im soziologischen Sinne normativ zu binden sucht.

Zugleich gerät Ideologie als Phänomen damit auch in die Nähe der Religion, und zwar so weit, daß Hayes in seiner letzten Abrechnung mit seiner lebenslangen Beschäftigung mit der Theorie des "Nationalismus" folgende Frage stellt, die seinem Buch auch den Titel gegeben hat: Nationalism - A religion? 16 Hayes meint Anlaß zu finden, diese Frage positiv zu beantworten 17 .

Er begründet dies etwa mit der Hervorhebung einzelner Menschen, Führer und Idole - seien es die Begründer der betreffenden Ideologie, seien es charismatische Führer - in die Rolle des Allwissenden und Allmächtigen, ihre Vergötterung oder Vergötzung durch kultische Handlungen (Bilder, Gedenkfeiern; strafrechtliche Sonderregelungen etc.) und Altäre (Mausoleen); mit Fahnenkult und Riten irrationaler Art, deren Nichtbefolgung mit schwe-

14 Auch der Begriff "sozialen Ideologie" könnte hier eingeführt werden, vgl. etwa Ralf Dreier, Recht - Moral - Ideologie, Frankfurt 1981, S. 159 f.: "Soziale Ideologien können als Systeme handlungsorientierter Vorstellungen definiert werden, die innerhalb von Gesellschaften oder Gruppen mehr oder weniger akzeptiert sind".

15 Dieses Motiv ist z.B. zu erkennen bei Aytekin Ataay, Kemâlizme [= Atatürkçü Ögretiye] Giris (Einführung in den Kemalismus [in die atatürkistische Lehre]), Gedenkschrift für Ümit Doganay I, Istanbul 1982, S. 159 ff.; Giritli (Fn. 5).

16 Carlton JH. Hayes, Nationalism: A Religion?, New York 1960.

17 Er tut dies für Nationalismus und Kommunismus (a.a.O., S. 14 ff.), aber die Feststellungen von Hayes lassen sich ohne weiteres auch auf andere Ideologien anwenden. Eine Variante hierzu entwickelt $H J$. Koppelmann, Nation, Sprache und Nationalismus, Leiden 1956, S. 116 ff. Vgl. vor allem auch Rainer Wittram, Nationalismus und Säkularisienung, Lüneburg 1949; Jürgen Gebhardt, Nationale Identität und nationale Ideologie, ZfP 1985, S. 237 ff.(245 f.). 
ren sozialen und strafrechtlichen Nachteilen verbunden ist ${ }^{18}$ - all dies mit Anspruch auf Ewigkeit. Damit trifft Hayes auch das Wesen des Kemalismus, wie er heute zwar abnehmend, aber dennoch weiter gepflegt wird, wo wie in einer Religion das Individuum bzw. der Staatsbürger ständig zum Bekenntnis herausgefordert, ja gezwungen wird19, das dann schnell zum Lippenbekenntnis entartet, wenn der Glaube fehlt; wo verdächtig ist, wer ihn zwar lebt, sich aber dem Dogmatismus verweigert20. Ähnlich wie in der Religion stellt sich auch die Frage nach der zuständigen Instanz mit der - wie auch immer legitimierten - Definitionsgewalt einschließlich der Befugnis zum Erlaß der "Durchfühnungsbestimmungen". Bei der Religion mag es die Kirche sein, im Marxismus ist es die Partei und beim Kemalismus ist es die Große Nationalversammlung zusammen mit der Hohen Atatürk-Gesellschaft für Kultur, Sprache und Geschichte als "Chefideologe". Wiederum Hayes selbst hat die Diagnose auch hinsichtlich des türkischen Nationalismus gestellt: "Nationalism became the official Turkish religion" 21 .

Damit befindet sich Hayes in Einklang mit der "Quintessenz" der Überlegungen von Gebhardt, der seinerseits Talmon zitiert: "Der Tod Gottes im 18. Jahrhundert schickte viele Völker auf die Suche nach einem Brennpunkt ihrer kollektiven Identität, die abgelöst war von der Kirche und der brüderlichen Gemeinschaft christlicher Gläubigkeit. Solch ein Ersatz wurde in der Nation gefunden."22. Für die Türkei sollte dieser Satz umformuliert werden:

"Der Rückzug Gottes aus der Politik, der Tod des osmanischen Sultanats und islamischosmanischen Kalifats schickte das osmanische Volk (nach dem 1. Weltkrieg das anatolische Volk) auf die Suche nach einem Brennpunkt ihrer kollektiven Identität, die abgelöst war von islamischen Institutionen und der bruderlichen Gemeinschaft islamischer Gläubigkeit. Solch ein Ersatz wurde in der Nation gefunden."

18 Hayes, a.a.O., S. $164 \mathrm{ff}$.

$19 \mathrm{Vgl}$. die in der Wochenzeitschrift Nokta v. 8.5.1988, S. 29 ff. referierte Kritik einiger modemer Kemalisten (z.B. Mete Tunçay, Taha Akyol, auch Süleyman Demirel).

20 Plastisch hierzu Aslan (Fn. 8), insbesondere S. 21 ff.

21 Hayes, a.a.O., S. 131.

22 Gebhardt, a.a.O., S. 246 (dort zit. JN. Talmon, The Myth of the Nation and the Vision of Revolution, London 1981, S. 1). Der "Tod Gottes" erscheint hier als Übertreibung, man wird die Formel als Rückzug von Religion und Kirche aus der Staatspolitik interpretieren müssen. 


\section{Der Nationalismus}

\section{Begriff}

Der Begriff des Nationalismus23 ist vom Begriff der Nation24 naturgemäß nicht zu trennen. Klassisch ist die Unterscheidung zwischen Staatsnation und Kulturnation oder auch ethnischer Nation. Staatswissenschaftlich und verfassungsrechtlich relevant ist meist nur der Begriff von der Staatsnation, soweit die Verfassung die Legitimation ihrer Institutionen von einem Souverän herleitet, dem "Volk" als Gemeinschaft aller Staatsbürger. Dennoch gibt es Verfassungen, die daneben den Begriff der Kulturnation rezipieren und der Zugehörigkeit zu einer Nation oder verschiedenen Nationalitäten rechtliche Bedeutung beimessen. Dies geschieht entweder, um den Status verschiedener Volksgruppen in einem Staatsverband zu bestimmen (z.B. Zyperns Verfassung von 1960, Belgien), oder aber um zu demonstrieren, $\mathrm{da}$ das Selbstverständnis des Staatsvolkes als Nation nicht an der Staatsgrenze aufhört (Deutschland). Als Rechtsfolgen dieses letzteren Verständnisses sind die direkte Gewährung der Staatsangehörigkeit für außerhalb des Staatsverbandes lebende Menschen denkbar oder aber die Besserstellung von Volksangehörigen durch Einbürgerungsprivilegien.

Für Frankreich wird ausschließlich die Geltung des Staatsnationsbegriffs angenommen 25 . Der "Franzose" wird nicht ethnisch definiert, sondern über seine Staatsangehörigkeit, die historisch - auf das Untertanenverhältnis gegenüber dem französischen König zurückgeht, ohne Rücksicht auf romanisch-keltische, westkeltische (bretonische), germanische oder baskische Wurzeln der ethnischen Zuordnung; mit der Französischen Revolution wurde die Untertanenschaft durch die Zugehörigkeit zur Nation ersetzt, die dann auch der Legitimierung des neuen Regimes zu dienen hatte.

Ein dritter Nationbegriff ist der religiöse, der sich wiederum in zweierlei Begriffe aufteilen läßt. Die Welt bzw. der Weltstaat des Islams erkennt seine "Staatsangehörigen" am Bekenntnis zu Allah und seinem Propheten Mohammed. Besonderen Rechtsverhältnissen

23 Vgl. einen allgemeinen Definitionsversuch bei Koppelmann, a.a.O., S. 175 ff.; s.a. Benjamin Akzin, State and Nation, London 1964, S. 46 ff.; Gebhardt, a.a.O. Vgl. auch die auf die heutigen Umbrüche bezogene Schrift von Panayotis Kondylis, Nationalismus zwischen radikalisierter Tradition und massendemokratischer Modemisierung, in: ders., Planetarische Politik nach dem Kalten Krieg, Berlin 1992, S. 59 ff;; H. Bartel/D. Fricke (Hrsg.), Wörterbuch der Geschichte Bd. II, Köln 1983, "Nationalismus", S. $734 \mathrm{f}$.

24 Auf die Einzelheiten des Streits um den Begriff der Nation soll hier nicht eingegangen werden. Vgl. Anthony D. Smith, Theories of Nationalism, London 1971, S. $186 \mathrm{ff}$.; Akzin, a.a.O., S. 29 ff.; Dieter Blumenwitz, Der Nationenbegriff und die deutsche Frage, ZfP 1985, S. 268 ff.; Wörterbuch der Geschichte, a.a.O., "Nation", S. 724 ff.

25 Vgl. dazu Theodor Veiter, Nation und Volk als Rechtsbegriffe unter besonderer Berücksichtigung der deutschen Frage, in: Staatliche und nationale Einheit Deutschlands - ihre Effektivität (hrsg. v. Dieter Blumenwitz und Boris Meissner), Bonn 1985, S. $105 \mathrm{ff}$. 
gegenüber dem islamischen Staat unterworfen, aber nicht seine "Staatsangehörigen" sind die Mitglieder der "Nationen" (millet), die im traditionellen Kernland des Islam, auf der Arabischen Halbinsel, ethnisch durchaus gleicher Herkunft sein konnten. Gemeint sind hiermit die vom Islam anerkannten Buchreligionen, deren Anhänger - im Gegensatz zu den Heiden - bestimmte "staatsbürgerliche" Rechte und Pflichten haben (dazu noch einmal unten V 9). Eine andere Variante eines religiös orientierten Nation-Begriffs ist der des Judentums oder der Armenier: Religion wird zu einem die ethnische Gemeinschaft einigenden Band, das sogar eine - wie bei den Armeniem im frühen Mittelalter - für Staatsgründungen ausreichende Kraft entfaltet. Der islamische Nationbegriff war auch derjenige des Osmanischen Reiches, in dem Christen und Juden seit jeher eine hierauf beruhende Rechtsstellung innehatten. Der Erwähnung wert ist an dieser Stelle, daß diese Verwendung des Nation-Begriffs auch der christlichen Welt bekannt war. Noch im Jahre 1836 wurde der "Nationalismus" als eine Lehre bezeichnet, wonach bestimmte Nationen von Gott auserwählt seien26. Die politische Funktion des religiösen Nation-Begriffs ist ursprünglich die gleiche wie bei den anderen Nation-Begriffen: er soll eine politische Einheit beschreiben, die durch ein gemeinschaftliches Bewußtsein zusammenhält. Dazu ist gerade eine gemeinsame Religion, ein gemeinsamer Glaube besonders geeignet. Nicht umsonst hat daher die Ideologisierung des säkularen Nation-Begriffs im Nationalismus sakrale Elemente.

Für den völkerrechtlichen Nation-Begriff schließlich sind - insoweit besteht Deckungsgleichheit mit der Staatsnation - die Staatsgrenzen ausschlaggebend27, er ist auf den französischen und englischen Nation-Begriff zurückzuführen28. Selbst dort also, wo die Kulturnation oder Glaubensnation über Staatsgrenzen hinausreicht oder weit hinter einer Staatsgrenze zurückbleibt, erlangt nur die Staatsnation Völkerrechtssubjektivität. Kulturnationale Einheiten sind bestenfalls Träger des Selbstbestimmungsrechts oder Minderheiten im Sinne des Art. 27 des UN-Paktes über die bürgerlichen und politischen Rechte29.

Unterschiedliche Verständnisse vom Begriff der Nation können dort, wo es verschiedene Nationalitäten im Sinne des Begriffs von der Kulturnation gibt, zu Konflikten führen. Während Deutschland, insbesondere zuletzt die Bundesrepublik Deutschland, wegen der in den Staatsgrenzen bestehenden weitestgehenden Deckungsgleichheit von Angehörigen der Staatsnation und Angehörigen der Kultumation kaum vor derartige Konfliktlagen gestellt

26 Indirekt zitiert bei Smith, a.a.O., S. 167.

27 Veiter, a.a.O., S. 107.

28 "United Nations" (Vereinte Nationen) ist keine Organisation der "Nationen" oder "Völker", sondem der Staaten.

29 Dieser Artikel steht mit einem Staatsnation-Konzept in problematischer Beziehung. Wie früher schon Frankreich (vgl. Manfred Nowak, UNO-Pakt über bürgerliche und politische Rechte und Fakultativprotokoll, CCPR-Kommentar, Kehl 1989, S. 802 f.) will die Türkei den Pakt ebenfalls nur mit dem Vorbehalt ratifizieren, daß unter Minderheit nur diejenigen zu verstehen seien, deren Schutz der Lausanner Vertrag von 1923 anordnet (vgl. Tageszeitung Cumhuriyet v. 23.7.1992). 
wurde $^{30}$, entwickelte sich zum Beispiel in Frankreich Anfang der siebziger Jahre ein "okzitanischer" und "bretonischer" Nationalismus, der sich zugunsten kulturnationaler Identitäten gegen das staatsnationalistische Machtmonopol in Paris richtete. Der auch in sporadische Gewalttätigkeiten ausbrechende Konflikt wurde sowohl infolge von Zugeständnissen aus Paris (z.B. die Nationalsprache als Unterrichtsfach in der Schule und Liberalisierung der Kulturpolitik) als auch wegen des geringen Rückhalts der militanten Separatisten in der Bevölkerung weitgehend beigelegt 31 . Hier wirkte sich der Umstand aus, daß die Staatsnation nicht "Abstammungsgemeinschaft" 32 ist und daher nicht auf emotionalverwandtschaftlichen Bindungen beruht, sondern vom gemeinsamen politischen Willen getragen ist, der sich - soll die Nation als solche erhalten bleiben - nur mit politischer Vernunft umsetzen läßt.

Wie unten noch gezeigt werden wird, folgt der kemalistische Nationalismus dem Begriff der Staatsnation 33 .

30 Konflike werden hier auf Verfeinerungen verlagert: Sie entstehen zwischen Stämmen (z.B. mit unterschiedlichen Dialekten und Volkstrachten) oder "Staatsnationen" auf Landesebene in willkürlichen Grenzen (Bayem besteht nur zu einem Teil aus "Bayem"; der Name Baden-Württemberg enthält noch nicht einmal einen Hinweis auf einen Stammesnamen). Vgl. im übrigen Helmut Rumpf, Die Nation in rechtlicher Sicht, in: Nation und Selbstbestimmung in Politik und Recht (Göttinger Arbeitskreis: Veröffentlichung Nr. 433), Berlin 1981, S. 7 ff.; Theodor Veiter, a.a.O.

31 Ein ähnlicher Konflikt schwelt derzeit in Korsika weiter. Dies ist wohl damit zu erklären, daß die Korsen sich wegen der vergleichsweisen kurzen Zugehörigkeit Korsikas zu Frankreich und damit fehlender Assimilierung ihre kulturelle Eigenständigkeit in sehr viel größerem Umfang erhalten haben.

32 Waldemar Mitscherlich, Volk und Nation, in: Handwörterbuch der Soziologie (hrsg. v. Alfred Vierkandt), 2. Aufl., Stuttgart 1959, S. 644 ff.

33 Nicht übemommen wird hier ein Versuch, den "europäischen" vom "türkischen" Nationalismus zu unterscheiden, und zwar auch begrifflich. Dies geschieht einem Vorschlag zufolge durch Verwendung von "nasyonalizm" und "milliyetçilik" - das eine eigentlich nur das türkische Synonym für das andere. Richtig ist daran nur, daß es sicherlich historische Unterscheidungsmöglichkeiten verschiedener Nationalismus-Bewegungen gibt. Das soziale und politische Faktum in einem umreißbaren politischen Zusammenhang ist jedoch immer wieder vergleichbar; gleiches gilt für den Weg von Nation und Nationalbewußtsein zur Ideologie, sei es die Ideologie einer gesellschaftlichen Gruppe oder des Staates. Der Begriff des Nationalismus ist daher universaler Nenner aller Nationalismus-Theorie. Ibrahim Kafesoglu, Atatürk Ilke'lerin Tarihî Temelleri (Die historischen Grundlagen der Prinzipien Atatürks), in: Kafesoglu/Saray (Fn. 8), S. 14 ff. etwa rechtfertigt mit einem solchen Versuch die Historiographie eines eigenen türkischen Nationalismus bis in die frühen Hunnenreiche zurück, auf der der kemalistische Nationalismus historisch beruhen soll. Konsequent wäre diese Unterscheidung nur, wenn Kafesoglu jedem einzelnen historisch und regional eingrenzbaren nationalen Phänomen einen eigenen Begriff zuordnen würde, vor allem wird Kafesoglu auch der Variantenvielfalt des europäischen Nationalismus in keiner Weise gerecht. Es geht ihm offenbar nur darum, der Möglichkeit einen Riegel vorzuschieben, daß die Verknüpfung des kemalistischen Nationalismus mit vergleichbaren früheren oder gleichzeitigen Phänomenen anderswo vermieden und die Einzigartigkeit des kemalistischen Nationalismus hervorgehoben wird. 


\section{Der ideologische Charakter des Nationalismus}

Nationalismus ist die ideologisierte (und damit im oben ausgefühnten Sinne sakralisierte) Vorstellung von und Identifizierung mit einer Nation, wobei heute insoweit der religiöse Nation-Begriff unbeachtet bleibt, da hier die Religion an sich die integrative und sakrale Funktion des Nationalismus übernimmt. Um den ideologischen Charakter zu entwickeln, der ihm begrifflich anhaftet, bedarf der Nationalismus neben dem Begriff der Nation eines Zwecks bzw. einer Idee. Zentral ist der Zweck der Einheit derjenigen Menschen, deren Gesamtheit die betreffende Nation ausmacht. Hier liegt es bereits auf der Hand, daß die Idee der Staatsnation (Einheit der Gemeinschaft der Staatsbürger) gegenüber der Idee der Kulturnation (Einheit der Gemeinschaft der Stammesbrüder und unter Umständen ihrer weiteren Verwandten) für einen gegebenen Staat politisch völlig verschiedene Folgen haben kann. Der Zweck der Einheit wird darüberhinaus besonders dort, wo emotionale Gründe sich nicht zu einem einigen Nationalbewußtsein entfalten können, mit rationalen Gründen untermauert werden. Wo also die Ideologie der Größe (Frankreich) oder von Blut und Boden (Deutschland) nicht ziehen kann, treten andere Gründe ein. Beim Kemalismus, der die Türkei aus einer Niederlage zu begleiten hatte, die als Stachel tief im Selbstbewußtsein der noch nicht sich selbst als solche begreifenden türkischen Nation 34 saß, war es das gemeinsame Überleben durch "Modemisierung", der Weg zu europäischer Zivilisation35. Vor allem seit 1980 wurde dann als weiteres ideelles Band die langfristige kulturelle Tradition entdeckt: die Tradition der großen Kulturen Anatoliens von den Hethitem über Byzanz und die Osmanen bis heute.

\section{Der türkische Nationalismus}

Aus der Vielfalt der möglichen Nationalismen erscheint für den modernen kemalistischen Nationalismus derjenige einschlägig, der sich auf eine Staatsnation bezieht und das ideelle Ziel der Modemisierung verfolgt, und zwar auf der Grundlage der Einheit der Gemeinschaft der Staatsbürger. Und um diese Einheit zu gewährleisten, wird dieser Nationalismus einigende Momente hervorheben müssen wie etwa eine gemeinsame Nationalsprache (die, anders als bei der Kulturnation, gerade nicht unbedingt die Muttersprache fast aller sein muß), eine einheitliche Kulturpolitik (die verschiedenen Kulturelementen zumindest ein gemeinsames Dach zu geben versucht, aber auch der Derogation oder Assimilation "fremder" Kulturelemente dienen kann) und eine Außenpolitik der nationalen Ehre. Auch das

34 Selbst die Bezeichnung "türkisch" war für die neue "Nation", die aus der in Anatolien und Osthhrakien lebenden Restbevölkerung des osmanischen Reiches bestand, neu und wenig gewohnt.

35 Hier drängt sich die Parallele zu den nationalistischen Entwicklungen in den neuen, aus der Sowjetunion hervorgegangenen Staaten auf: vgl. Panayotis Kondylis, Nationalismus zwischen radikalisierter Tradition und massendemokratischer Modemisierung, in: ders., Planetarische Politik nach dem Kalten Krieg, Berlin 1992, S. 59 ff. 
emotional-moralische Moment der Brüderlichkeit wird als Integrationsfaktor betont werden müssen.

\section{a) Die Entwicklung des türkischen Nationalismus}

Die Ideen des europäischen Nationalismus ergriffen im letzten Jahrhundert nicht nur die Balkanvölker, sondern auch die osmanische Elite in den Großstädten. Den durch die christlichen Westmächte geförderten kulturnationalen Separatismusbestrebungen versuchte zunächst eine Gruppe von Intellektuellen und der politischen Elite gegen Ende der sechziger Jahre des letzten Jahrhunderts eine staatsnationale Staatsauffassung entgegenzusetzen (Jungosmanen) ${ }^{36}$, die in der ersten modernen Verfassung im Jahre 1876 ihren Niederschlag fand. Dieses Gedankengut einer "staatsnahen" Elite war jedoch nicht geeignet, die auseinanderdriftenden Nationalbewußtseinslagen verschiedener Völkerschaften im osmanischen Reich - seien es die Araber, seien es die Balkanvölker - in einem gesamtheitlichen "Staatsnationalbewußtsein" bei gleichzeitiger Wahrung kultureller und ethnischer Vielfalt zusammenzuführen. Das kultumationale Element dieser Bewegung entwickelte jedoch zu Beginn dieses Jahrhunderts Tendenzen eines "türkischen" Nationalismus37. Die "linke" Partei Union und Fortschritt der Jungtürken, der im Jahre 1908/1909 der Sturz von Abdülhamit II. gelang, ist in diese Tendenz einzuordnen. Dies schloß zwar das friedliche Zusammenleben verschiedener Nationalitäten unter dem Dach des Osmanischen Reiches nicht aus - zumal sich der Sultan nach wie vor als Herrscher aller Osmanen verstand -, erhöhte jedoch infolge des Hinzutretens rassistischer Tendenzen die Konfliktträchtigkeit erheblich und vernachlässigte bereits die spezifisch "osmanische" Tradition mit ihrer Besinnung auf die Leistungen der osmanischen Dynastie, die seit Gründung ihres Reiches in Bursa (Brussa) für die "Einheit in der Vielfalt" des osmanischen Reiches stand.

Eine weitere Strömung fußte auf einer pantürkischen Idee und hatte die Vereinigung aller türkischen, mehr noch: turanischen Völker zum Ziel (Turanismus)38. Diese Variante stellte sowohl in den Augen ihrer Kritiker als auch ihrer Verfechter eine Utopie dar. Auch heute

36 Vgl. zu den Jungosmanen Bernard Lewis, The Emergence of Modem Turkey, London 1961, S. 147 ff.; Ihsan Sungu, Tanzimat ve yeni osmanlilar (Die Reformperiode und die Jungosmanen), in: Tanzimat (Die Reformperiode) I, [eine in Istanbul 1940 vom Kulturministerium herausgegebene Festschrift, deren zweiter Band nie erschienen ist], S. 777 ff.; David Kushner, The Rise of Turkish Nationalism 1876-1908, London/Totowa 1977, S. 7, 37 ff.; ErikJan Zürcher, The Unionist Factor, Leiden 1984, S. 1 ff.

37 Vgl. Kushner, S. 97 ff. Leitende Figuren des "Türkismus" waren Yusuf Akçura (siehe dazu François Georgeon, Türk Milliyetçiligin Kökenleri (Die Wurzeln des türkischen Nationalismus) Yusuf Akçura [1876-1935], Ankara 1986) und Ziya Gökalp (vgl. Ziya Gökalp, The Principles of Turkism, übers. und komm. v. Robert Devereux, Leiden 1968).

38 Dazu ausführlich Zarevand, United and Independent Turania, Leiden 1971 (aus dem arnnenischen Original [Boston 1926] übers. v. VN. Dadrian). 
gibt es Anhänger dieser Idee, die infolge des Zusammenbruchs der Blöcke in Ost und West und der damit verbundenen Notwendigkeit einer Neuorientierung der Türkei ungeahnte Aktualität gewonnen hat.

Die Rechtsnachfolge des zusammengebrochenen Vielvölkerstaates wurde im Jahre 1921 durch einen türkischen Rumpfstaat angetreten, der nur noch über das anatolische Territorium und einen kleinen Teil Thrakiens verfügte. Großangelegte Aktionen des Bevölkerungsaustausches mit den Balkanländern führten zum Auszug vor allem der christlichen Bevölkerungsteile, Uberreste erhielten sich unter dem Schutz des Lausanner Vertrages in Istanbul und im Osten Anatoliens. Neben einigen ihrer Zahl nach unbedeutenden Bevölkerungsteilen (Laz, Turkmenen, Araber u.a.) blieben als wesentliche kulturnational definierbare Volksgruppe die Kurden übrig. Sie machten die Entwicklung eines ethnischen Nationalismus unmöglich39.

\section{b) Das kemalistische Nationalismus-Konzept}

Der Minimierung eines hierin liegenden Konfliktpotentials konnte, wollte man die Einheit von Staatsgebiet und Staatsvolk sicherstellen, zweierlei dienen: die Errichtung eines Bundesstaates oder die Errichtung eines Zentralstaates, der den Interessen der verschiedenen Bevölkerungsgruppen gerecht zu werden oder aber sie zu assimilieren suchte.

Das - im Verlauf der Gründungsphase und weiteren Geschichte der jungen Republik keineswegs einheitliche 40 - kemalistische Nationalismus-Konzept gilt der Variante des Zentralstaats, ohne sich zwingend entweder für die Berücksichtigung der Gruppeninteressen oder für strikte Assimilation zu entscheiden. Ausgangspunkt war jedenfalls - ähnlich wie in Frankreich - das Verhältnis des der Staatsgewalt Unterworfenen zum Staat, die Staatsangehörigkeit: Türke ist, "den mit dem Türkischen Staat das Band der Staatsangehörigkeit verbindet" (Art. 66 Abs.1 TV 1982). Damit allein läßt sich jedoch das nötige Bewußtsein der Zusammengehörigkeit in einer Nation nicht herstellen.

Der integrative islamische Nationbegriff sollte mit der Ausrufung der Republik und mit der danach einsetzenden Laizisierung von Staat und Gesellschaft wegfallen. Die Abschaffung

39 Dies hatte man schon in der Nationalversammlung von 1920 erkannt, wo man in einer heftigen Debatte um die nationale Identität stritt. Türkistischem wurde der Vorschlag entgegengesetzt, sich auf "Osmanen" zu einigen, Mustafa Kemal Pascha beendete die Debatte mit einer Gleichsetzung aller islamischen Elemente der ethnisch aufgesplitterten Bevölkerung unter dem Begriff "Türke" (siehe Hiff zi V. Velidedeoglu, Ilk Türkiye Büyük Millet Meclisi'nde Atlatilan Etnik Sorun (Die überwundene ethnische Frage in der ersten Großen Nationalversammlung der Türkei), Tageszeitung Cumhuriyet v. 12.6.1988, S. 2).

Vgl. vorstehende Fn. 
des zu Beginn des Befreiungskampfes 1919 noch kaum in Frage gestellten Sultanats bedeutete das Ende der Herrschaft eines osmanisch-islamischen Universalismus und Multinationalismus. Die Abschaffung des Kalifats 1924 und schließlich des Islam als Staatsreligion 1928 suchte auch der möglichen Idee eines islamischen Nation-Begriffs den Boden zu entziehen, obwohl sie doch gleichzeitig durch den Friedensvertrag von Lausanne zugunsten der christlichen und jüdischen Minderheiten festgeschrieben worden war.

Offen blieb dagegen zunächst das Verhältnis des Kemalismus zu ethnischen Identifizierungsversuchen im Sinne eines Kulturnation-Begriffs. Die Eigenständigkeit der Kurden war ein politisches Problem, jedoch kein kulturelles. Die türkische Delegation in Lausanne ging genauso von der ethnischen und kulturellen Eigenständigkeit der Kurden aus wie Atatürk selbst, der ursprünglich nicht einmal von der "türkischen Nation", sondern von der "Nation der Türkei" gesprochen hatte 41 .

Die noch bei den Lausanner Verhandlungen von der türkischen Delegation unter Ismet Inönü vertretene Fiktion der Schicksalsgemeinschaft der Türken und der Kurden und der Loyalität der Kurden gegenüber der Zentralregierung in Ankara wurde im Jahre 1925 durch den Aufstand Seyh Saids erschüttert. Hier dürfte wohl der Anfang einer spezifischen Kurdenpolitik anzusiedeln sein, die sich zunehmend als Assimilierungspolitik herausstellte. Mit der kemalistischen Nationalismus-Idee hat dies aber nichts zu tun. Die Politik mag richtig oder falsch gewesen sein - in jenem Zeitpunkt wurde die Existenz einer Ethnie der Kurden nicht bestritten. Entscheidend im Sinne des kemalistischen Nationalismus war ihre Staatsangehörigkeit, die rechtlich nichts mit Assimilation oder einer rechtlichen Fiktion der ethnischen und kulturellen Gleichartigkeit von Türken und Kurden zu tun hatte, wie sie dann später vertreten wurde.

Gegen diese Offenheit spricht auch nicht, daß Atatürk einmal das "Türkentum" als Grundlage des Nationalismus und der Republik bezeichnet hat 42. Er sah das, wenn man die einschlägigen Zitate richtig einordnet, nämlich vor allem im Zusammenhang mit dem Problem der multinationalen Struktur auch der Bevölkerung des Reststaates, die ein gemeinsames Nationalgefühl zu entwickeln hatte. Der einzige Begriff - wollte man die gemeinsame Zugehörigkeit zum Islam nicht kontraproduktiv betonen -, der der Bevölkerung plausibel gemacht werden konnte, war eben derjenige des "Türkentums" - ein "kultur"-nationaler Identifizierungsversuch, der erst noch zum Erfolg geführt werden mußte. Das subjektive Zusammengehörigkeitsgefühl, das für den Nationalismus charakteristische integrative subjektive Element, das Nationalgefühl, mußte erst noch geschaffen werden. Dazu diente einerseits das Strafrecht - z.B. mit Hilfe einer Vorschrift, die die "Propaganda gegen die

41 Vgl. Seraffetin Turan, Atatürk Milliyetçiligi (Der Nationalismus Atatürks), Belleten LI/204 (Nov. 1988), S. 849 ff. (853 f.).

42 Vgl. Kafesoglu, S. 60. 
nationalen Gefühle" verbot (Art. 142 Abs.3 türk.StGB ${ }^{43}$ ); andererseits spielten die von Atatürk eingerichtete Türkische Sprachgesellschaft und die Türkische Geschichtsgesellschaft eine zentrale Rolle44, die die gemeinsame Sprache fast völlig neu zu entdecken und zu entwickeln und die türkische Geschichte insbesondere unter dem Blickwinkel des Unabhängigkeitskampfes mit seinem solidarisierenden Effekt neu zu schreiben hatten, um das zu unterfüttern, was als Türkentum im kemalistischen Sinne erst noch Konturen zu gewinnen hatte. Man sollte also Atatürks Nationalismus nicht unbesehen in die Tradition eines Nationalismus von Ziya Gökalp stellen, der zwar politischer, nicht aber pragmatischer Natur war.

Der pragmatische kemalistische Nationalismus allein hat also durchaus Raum für kulturnationale Identifizierungen als Teile einer gesamttürkischen Identität.

Trotz des theoretischen Ansatzes zu einem pragmatischen Nationalismus kam es zu Lebzeiten Mustafa Kemal Atatürks zu gesetzgeberischen Aktivitäten, die auf eine Assimiliation andersartiger Kulturelemente hinausliefen. So ermöglichte ein Gesetz aus dem Jahre 1934 die Zwangsumsiedlung solcher Bevölkerungsgruppen, die nicht der "nationalen Kultur" verbunden waren. Hier zeigte sich, jedenfalls in der Praxis, ein kultumationales Element im Nationalismus-Begriff der dreißiger Jahre, das bis heute die Möglichkeit einer klaren, rationalen Linie des kemalistischen Nationalismus-Konzepts vereitelt. Ähnlich verlief auch die Forschungspolitik der Türkischen Geschichtsgesellschaft, die auf die Erforschung der Geschichte der Kurden als wichtigster ethnisch nichttürkischen Gruppe verzichtete.

Dieser von einem pragmatischen Nationalismus-Konzept in manchem wichtigen Punkt abweichenden Variante des kemalistischen Nationalismus folgt die türkische Verfassung mit ihrer spezifischen Ideologie, die man als "postkemalistisch" bezeichnen könnte.

\section{Der ideologische Gehalt der türkischen Verfassung}

\section{Allgemeines}

Der ideologische Charakter eines Rechtssatzes oder einer Ansammlung von Rechtssätzen ergibt sich nicht nur und nicht immer aus dem Wortlaut des Rechtssatzes selbst, sondern häufig auch aus der Art und Weise der Formulierung und des Einsatzes bestimmter Begriffe im Textzusammenhang. So drückt die Phrase von der unteilbaren Einheit von

Vgl. Abdullah P. Gözübüyük, Türk Ceza Kanunu (Türkisches StGB [Kommentar]) Bd. II, 5. Aufl. Istanbul 1990, zu Art. 142; Erol Cihan, Millî Duygulara Aykìrì Propaganda Yapma Suçu (TCK. m. 142/b.3) (Die Straftat der Propaganda gegen die nationalen Gefühle [Art. 142 Abs. 3 türk.StGB]), IÜHFM XLIII (1-4), S. 45 ff.

44 Vgl. dazu z.B. Ernst E. Hirsch, Die kemalistische Türkei, in: Mustafa Kemal Atatürk (Fn. 6), S. 25 ff. 
Staatsgebiet und Staatsvolk bei isolierter Betrachtung lediglich ein Element der Staatsraison aus, weist auf legitime Herrschaftssicherung hin. Unverdächtig erscheint auch, wenn "die Sprache" des Staates Türkisch sein soll oder von nationalem Interesse die Rede ist. Den ideologischen Charakter erhalten solche nachher in einem ideologischen Begriff wie dem des "Nationalismus" zusammengefaßten Elemente aber zum einen durch Institutionalisierung von Kultur in einer verfassungsrechtlich eigens abgesicherten Institution (Hohe Atatürk-Gesellschaft) und zum andern vor allem durch "ideologisierende" Elemente, wie sie in der Präambel zu finden sind: schmückende Begriffe, die durch ihre interpretatorische Verknüpfung mit den eben genannten Rechtsbegriffen ungeahnte Gewalt entfalten.

Anders als das Grundgesetz für die Bundesrepublik Deutschland oder die Verfassung der italienischen Republik, die in mancher Hinsicht der türkischen Verfassung von 1961 Pate gestanden haben, deren Grundstrukturen auch in der türkischen Verfassung von 1982 wiederzuerkennen sind, begnügt sich die türkische Verfassung nicht mit einem Bekenntnis zur Demokratie und zum Rechts- und Sozialstaat. Abgesehen davon, daß das Prinzip der Freiheitlichkeit (in dubio pro libertate) dem Prinzip des Vorrangs der Staatsautorität (in dubio pro auctoritate) hat weichen müssen 45 , beruht die türkische Verfassung seit spätestens 1937 auf ideologischen Grundlagen, die ein spezifisches Konzept von Staat und Nation beinhalten, das - wird diesen Grundlagen streng gefolgt - erheblich enger ist als dasjenige eines modernen Staates, der nach dem Muster einer westlichen Demokratie im bürgerlichen Gesetzesstaat des späten 19. Jahrhunderts und in der Idee des liberalen Rechtsstaates verwurzelt ist. Diese ideologischen Grundlagen, die Staatsideologie der türkischen Verfassung wird im Kemalismus definiert.

Der ideologische Gehalt der türkischen Verfassung mag über die Zeit seit der Gründung der Republik wenig konstant gewesen sein. Nach der Aufnahme der "sechs Pfeile" im Jahre 1937 in die Verfassung von 1924 und damit der verfassungsrechtlichen Verfestigung der kemalistischen Staatsideologie ist es mit der liberalistischen Verfassung von 1961 zunächst wieder zu einer Abschwächung gekommen, die vielleicht gerade dem dynamistischen Element des Kemalismus Rechnung getragen und damit dem politischen Vermächtnis Atatürks am ehesten entsprochen hat. Eine Verstärkung ideologischer Züge, für die der bereits erwähnte Begriff "postkemalistisch" aufgegriffen werden kann, läßt sich dann wieder in der Verfassung von 1982 erkennen.

45 Vgl. dazu Bülent Tanör, Der Verfassungswandel in der Türkei, in: Die Türkei im Umbruch (hrsg. v. Yildirim Dagyeli und Ibrahim H. Özak), Frankfurt 1989, S. 11 ff. 
Für die Untermauerung dieser These bietet sich in erster Linie Art. 2 TV 1982 an, den auch das Verfassungsgericht immer wieder bei der Prüfung der Verfassungsmäßigkeit von Gesetzen heranzieht. Art. 2 TV 1982 lautet:

"Die Republik Türkei ist ein im Geiste des Wohles der Gemeinschaft, der nationalen Solidarität und der Gerechtigkeit die Menschenrechte achtender, dem Nationalismus Atatürks verbundener und auf den in der Präambel verkündeten Grundprinzipien beruhender demokratischer, laizistischer und sozialer Rechtsstaat".

Unmittelbar zum Ausdruck kommen hier als kemalistische Prinzipien vor allem der Nationalismus (zugleich in der Formel der nationalen Solidarität) und der Laizismus als normative Grundlagen der türkischen Verfassungsordnung 46 . Zu beachten ist aber vor allem auch der Verweis auf die in der Präambel niedergelegten Grundsätze.

\section{Die Präambel}

a) Die Verfassung von 1924 enthielt überhaupt keine Präambel. Der Grund hierfür liegt darin, daß die Verfassung von 1924 in einer Zeit entstanden ist, als die Leistungen der militärischen, politischen und Staatselite mit Mustafa Kemal Atatürk an der Spitze für sich sprachen: Zwei Jahre zuvor war der Unabhängigkeitskrieg gewonnen, ein Jahr zuvor waren die Verhandlungen zum Friedensvertrag von Lausanne mit überraschendem Erfolg abgeschlossen worden. Die Macht der Großen Nationalversammlung der Türkei war absolut, es bedurfte keiner Rechtfertigung des bisher Geleisteten. Es bedurfte keiner Beteuerungen guten Willens und keiner emphatischen Appelle an die Anwender der Verfassung.

Anders war es im Jahre 1961. Das Militär hatte ein demokratisch gewähltes parlamentarisches Regime mit dem Ziel gestürzt, "den wahren Rechtsstaat zu errichten". Nicht nur gegenüber den befreundeten Staaten in Europa, sondem auch gegenüber dem eigenen Volk, das an diesem Staatsstreich nicht beteiligt gewesen bzw. von dem nur ein Teil durch die am Putsch beteiligte Republikanische Volkspartei repräsentiert war, gab es Begründungs- und Legitimationsbedarf. Die Notwendigkeit der "besseren" neuen Verfassung war gegenüber der "versagenden" alten Verfassung hervorzuheben. Der Verrat der - demokratisch gewählten - Anwender der alten Verfassung konnte nur delegitimiert werden, wenn man dem Staatsstreich und damit der neuen Verfassung eine neue und überzeugende Legitimationsgrundlage gab. Dies geschah in der Präambel, die vor allem die Idee des Nationalis-

46 Zum Laizismus vgl. Christian Rumpf, Das Laizismus-Prinzip in der Rechtsordnung der Republik Türkei, JöR 36, S. 179 ff. 
mus hervorhebt und so insgesamt den eher liberalistischen Charakter der Verfassung von 1961 ideologisch relativiert, indem in einer an ein Glaubensbekenntnis erinnernden Weise formuliert wird47:

"Die Türkische Nation,... begeistert und beseelt vom türkischen Nationalismus, der alle einzelnen, Schicksals genossen in Glanz und Elend, zu einem unteilbaren Ganzen um das nationale Bewußtsein schart und das Ziel verfolgt, unsere Nation als gleichberechtigtes Mitglied der Völkerfamilie der Welt im Geiste nationaler Einheit ständig zu erhöhen..."

Im Zusammenhang mit der Erörterung des Nationalismus in der Verfassung wird darauf noch einmal zurückzukommen sein (unten V 2).

b) Die Präambel von 1982 übertrifft diejenige von 1961 bei weitem. Infolge der Rechtsprechung des Verfassungsgerichts, das die Präambel gemäß Art. 176 TV 1982 als integralen Bestandteil des Verfassungstextes zu begreifen und daher auch zur Auslegung anderer Verfassungsvorschriften heranzuziehen hat, strahlt sie mit ihrem Inhalt auch stärker auf die übrige Verfassung und ihre Interpretation aus, obwohl die Präambel kaum als eigenständige Norm zur Auslegungshilfe taugt48. So erfahren in der überlangen Präambel49 die kemalistischen Prinzipien eine pathetische Überhöhung ohnegleichen. Man findet einige der jenigen Merkmale, die den Nationalismus bzw. den Kemalismus als Ideologie in ihrem sakralen Charakter enthüllen. So ist etwa die Rede von "der Auffassung vom Nationalismus, wie sie Atatürk, der Gründer der Republik Türkei, der unsterbliche Führer und einzigartige Held, verkündet hat, und im Sinne Seiner (sic) Reformen und Prinzipien ..."; von "den Prinzipien und Reformen sowie dem Zivilisationismus Atatürks", dem "Prinzip des Laizismus". Außerdem haben die Streitkräfte durch den Putsch vom 12. September 1980 das ewige Türkische Vaterland und die Existenz des heiligen Türkischen Staates gerettet; die Verfassung wurde der Liebe der der Demokratie innig verbundenen Kinder der Nation übergeben, um sie in der Idee, dem Glauben und der Entschlossenheit anzuwenden und zu pflegen, verschiedenen Grundsätzen zu folgen; ewiges Dasein der Republik; materielles und geistiges Glück; nationaler Stolz, nationale Freude, nationales Leid - deutlicher ließe sich kaum der Zusammenhang zwischen Religion und Ideologie illustrieren.

47 Überarbeiteter Text aus E. E. Hirsch, Die Verfassung der Türkischen Republik (Staatsverfassungen derWelt, Bd. 7), Frankfurt 1966.

48 Vgl. zuletzt das "Kopf tuch-Urteil", auf deutsch abgedruckt in EuGRZ (Europäische Grundrechte Zeitschrift) 1990, S. 146 ff.

49 Vgl. die Übersetzung der türkischen Verfassung durch Ernst E. Hirsch, JöR 32, S. 507 ff.; Christian Rumpf, Beiträge zur Konfliktforschung 1983, S. $111 \mathrm{ff}$.; ders. im Anhang bei R. Geffken, Die Demokratie der Militärs, Köln 1983. 


\section{Der übrige Verfassungstext}

Die in der Präambel beschworenen Prinzipien Atatürks lassen sich auch im übrigen Verfassungstext ausmachen. Der Laizismus findet sich u.a. in Art. 136 TV 1982 wieder, der die Einrichtung des Präsidiums für Religionsangelegenheiten regelt50 oder in Art. 174 TV 1982, der acht "Reformgesetze" aus den zwanziger und dreißiger Jahren unter besonderen verfassungsrechtlichen Schutz stellt. Etatistische Elemente finden sich in den Vorschriften über die Wirtschaftsverfassung, populistische Elemente können mit dem Demokratieprinzip in Verbindung gebracht werden. Und während die Verfassung von 1961 als solche hätte als Konkretisierung des revolutionären Reformismus angesehen werden können, läßt sich dieser Grundsatz nur noch in den eben erwähnten Reformgesetzen erkennen, die infolge ihres Alters nichts Revolutionäres mehr an sich haben.

Insgesamt findet sich hier also eine spezifische ideologische Grundlegung der türkischen Verfassung, deren zentrales Element - neben dem vielleicht gleichwertigen Laizismus - der Nationalismus ist.

\section{Der Nationalismus in seiner verfassungsrechtlichen Ausgestaltung 51}

\section{Allgemeine Bemerkungen zur türkischen Verfassung}

Verfassung ist nicht nur der Verfassungstext, sondern das gesamte normative Gefüge, das einer Staats- und Gesellschaftsordnung ihr Gepräge gibt. Grundaussagen, die im Verfassungstext mit normativer Kraft getroffen worden sind, sollten sich auf allen Ebenen des Rechts - im Gesetz, in der Verordnung, im Erlaß - wiederfinden (Prinzip der Einheit der Rechtsordnung; Grundsatz der Rechtssicherheit). Sonst wäre das Tätigwerden des Verfassunggebers überflüssig und das Grundgesetz totes Recht, ein rechtlich bedeutungsloses Textgerippe.

Tatsächlich weist die türkische Verfassung von 1982 in einzelnen Zusammenhängen in dieser Hinsicht Unzulänglichkeiten auf. Dies ist nicht zuletzt deshalb der Fall, weil Übergangs-Art. 15 TV 1982 eine Vielzahl von Gesetzen der Kontrolle des Verfassungsgerichts entzogen hat, die während der Übergangszeit zwischen dem 12. September 1980 und Anfang Dezember 1983 vom Nationalen Sicherheitsrat der Übergangszeit erlassen worden sind und die gegenwärtige Staats- und Gesellschaftsordnung in einer Weise bestimmen, die

50 Näher zu dieser Institution Christian Rumpf, Das Präsidium für Relgionsangelegenheiten, ZfTS $1 / 89$, S. $21 \mathrm{ff}$.

51 Vgl. dazu auch Akin Düren, The Reforms of Atatürk in the Constitutional System and Nationalism, in: Papers and Discussion (Fn. 8), S. $171 \mathrm{ff}$. 
mit dem Verfassungstext nicht immer zu vereinbaren ist ${ }^{52}$. Dies gilt für Regelungen des Ausnahmezustandes, der politischen Parteien, der Gesetzgebung im Zusammenhang mit der Arbeitswelt, des Demonstrations- und Vereinigungsrechts.

Was indessen unser Thema angeht, so genügt für die Darstellung der rechtlichen Relevanz der kemalistischen Nationalismus-Konzeption und ihres - auch im Recht sichtbaren - ideologischen Charakters der Blick auf den Verfassungstext. Im nachfolgenden geht es daher nur um eine auf dem Text der Verfassung von 1982 beruhende Darstellung mit vergleichenden Hinweisen auf die Verfassungen von 1924 und 1961 vereinzelten Bezugnahmen auf einfaches Gesetzesrecht.

\section{Noch einmal: Die Präambel}

Die Funktion der Präambel für die Bestimmung des ideologischen Gehalts der türkischen Verfassung ist bereits erläutert worden. Dies soll noch einmal spezifisch für den Nationalismus aufgegriffen werden.

Im Text der Präambeln von 1961 und 1982 werden Verfassung und Nation miteinander in engen Zusammenhang gebracht. Verfassung ist das normative, rechtliche Gerüst der Staatsund Gesellschaftsordnung; Nation ist als ideelles Element sozial und kulturell integrierender Faktor der Staats- und Gesellschaftsordnung und zugleich Grundlage und Rechtfertigung ihrer Einheit. Es ist - wie die Verfassung als solche - zugleich Faktor für die Abgrenzung nach außen.

a) Dies wurde schon in der oben insoweit bereits zitierten Präambel der Verfassung von 1961 ideologisierend gestützt, indem wesentliche Begriffe der Nationalismus-Theorie fielen: Einheitsgedanke, Nationalbewußtsein, Souveränität, Gleichberechtigung in der internationalen Staatengemeinschaft. Das spezifisch "Ideologisierende" an dieser Präambel war aber hier nicht der auch für heutige Verhältmisse noch selbstverständliche Hinweis auf die Nation als materieller Gegenstand und Subjekt des Staates, sondern die emphatische Verwendung und Einsetzung nationalistischer Begrifflichkeit und ihr Appellcharakter - die Präambel als Glaubenssatz. Der "türkische Nationalismus" wurde als Triebfeder auf dem Weg zur Größe dargestellt, die das türkische Volk im Schoße der Völkerfamilie erlangen soll. Und schließlich ist noch die (oben nicht wiedergegebene) Erhöhung eines Menschen

52 Zur Fortgeltung des Übergangs-Art. 15 hatte das türkische Verfassungsgericht in den letzten Monaten des öfteren Stellung zu nehmen, wobei es feststellte, daß es keine Möglichkeit habe, entgegen Übergangs-Art. 15 TV die hierdurch geschützten Gesetze für verfassungswidrig zu erklären (vgl. z.B. Urt. v. 3.7.1991, E.1991/6, K.1991/20, in: RG Nr. 21165 v. 8.3.1992 sowie das in Fn. 65 zitierte Unteil); vgl. dazu Bülent Tanör, Iki Anayasa 1961/1982 (Zwei Verfassungen), Istanbul 1986 in toto. 
zu erwähnen, nämlich Atatürks, dessen in Form der Großschreibung der ihn bezeichnenden Pronomina (Er, Sein) gedacht wird - ein Stil, den man aus der Bibel und Texten in Bezug auf Gott, vielleicht noch auf Monarchen kennt.

b) Wie schon überhaupt mit ihrem ideologischen Gehalt geht auch bezüglich des Nationalismus die Präambel von 1982 noch erheblich weiter. Zunächst wird die "Nation" erhöht, das Türkische Volk: Die Verfassung wird durch "Es" gegeben, und zwar "in der Idee, dem Glauben und der Entschlossenheit, daß entsprechend der Auffassung vom Nationalismus" Atatürks ... "keine Meinung und Ansicht gegenüber türkischen nationalen Interessen, dem Grundsatz der Unteilbarkeit von türkischem Dasein, Staat und Staatsgebiet, den geschichtlichen und geistigen Werten des Türkentums und dem Nationalismus ... Atatürks werde geschützt [und] jeder türkische Staatsbürger ... von seiner Geburt an das Recht und die Möglichkeit habe, innerhalb der nationalen Kultur-, Zivilisations- und Rechtsordnung ein würdiges Leben zu führen, [und] die türkischen Staatsbürger in nationalem Stolz, in nationaler Freude und nationalem Leid, in ihren Rechten und Pflichten gegenüber dem nationalen Dasein, in Segen und Mühsal sowie in jeglicher Manifestation des Nationallebens geeint seien" ...

Hier wird nicht nur ein mehr oder weniger ausfüllungsbedürftiger Nationalismus Atatürks, sondern werden auch die geistigen und kulturellen Werte des Türkentums sowie die nationale Kultur und Zivilisation, die emotionale Gemeinschaftlichkeit in Freud und Leid innerhalb der "Nation" hervorgehoben; das Individuum des Bürgers wird auf das "Nationale" seines sozialen Wesens eingeschworen, ja sogar sein Persönlichkeitskern, seine Würde, in die Grenzen nationaler Kultur und Zivilisation verwiesen: der Nationalismus als Unterwerfung des Bürgers unter "nationalism, a religion" ... Einzelne Motive hieraus finden sich auch im übrigen Verfassungstext.

\section{Die Grundlagen der Republik}

Die Verfassungen von 1924, 1961 und 1982 haben sämtlich die Grundlagen der Republik in jeweils ihrem Art. 2 normiert.

a) Eine markante Metamorphose hat nur Art. 2 TV 1924 durchgemacht, der in der ursprünglichen Fassung den Islam zur "Religion des türkischen Staates" gemacht hatte. Symptomatisch ist schon fast, daß diese Metamorphose 1937 mit der Übernahme der sechs Pfeile des Kemalismus endete, nachdem seit 1928 jede entsprechende Grundlagenregelung gefehlt hatte. Selbstverständlich war es, daß mit den übrigen kemalistischen Prinzipien auch der Nationalismus aufgenommen wurde:

"Der türkische Staat ist ... nationalistisch ...". 
b) Mit der Verfassung von 1961 wurde daraus ein "... nationaler ... Rechtsstaat". Das ideologisierende Element in "nationalistisch" wurde ausgespart. Daß der Begriff "national" überhaupt in den Text aufgenommen wurde, war Ergebnis längerer Debatten der Repräsentantenversammlung, die in der Ubergangszeit seit Ende 1960 wesentlich an der Verfassunggebung beteiligt war.

c) 1982 kehrte sich die rückläufige Tendenz wieder um. Es waren die Ereignisse vor dem 12. September 1980, die den Verfassunggeber bewogen, das ideologische Element zu beschwören, in der Hoffnung, die integrierende Funktion der Ideologie und insbesondere des Nationalismus fruchtbar zu machen. Gleich mehrfach wurde in Art. 2 TV 1982 (siehe oben IV den Text) das nationalistische Element eingebracht und betont: nationale Solidarität - das subjektiv integrative Element; der Nationalismus Atatürks - das ideologisch-kemalistische Element; das gleiche noch einmal verstärkt über den Verweis auf die Grundprinzipien in der Präambel. Der Begriff des Rechtsstaats wird nicht nur mit den Attributen demokratisch und sozial versehen, sondem auch in das Korsett der (post-)kemalistischen Ideologie gezwängt. Der sich hier fortsetzende Geist der Präambel wird dann im Verlauf des Verfassungstextes weiter konkretisiert.

\section{Die unteilbare Einheit von Staatsgebiet und Staatsvolk53}

Diese Formel, wie sie auch in der französischen Verfassung zu finden ist, kann man als materiales Element des Nationalismus-Prinzips bezeichnen. In der Verfassung von 1924 war sie noch nicht enthalten, nicht einmal in den Eidestexten für die Abgeordneten oder den Präsidenten der Republik. Sie taucht erst in Art. 3 TV 1961 auf. Von dort wurde sie in Art. 3 TV 1982 übernommen. In beiden Fassungen ist sie mit der Anordnung "Türkisch als Amtssprache" (1961) bzw. "Türkisch als Sprache des Staates" (1982) verbunden.

a) In der Verfassung von 1961, und zwar erst in der Fassung nach der großen Verfassungsreform im Jahre 197154, erscheint sie als allgemeine Grundrechtsschranke in Art. 11 Abs.1 TV 1961 und immer wieder als Schranke einzelner Grundrechte wie in Art. 22 Abs.3 TV 1961 (Pressefreiheit) und anderen Grundrechten (z.B. Vereinigungsfreiheit Art. 29 TV

53 Vgl. dazu Sulhi Dönmezer, Devletin Ülkesi ve Milleti ile Bütünlügü ve Bölünmezlik Ilkesi (Der Grundsatz der unteilbaren Einheit von Staatsgebiet und Staatsvolk), in: Cumhuriyet Döneminde Hukuk - Istanbul Üniversitesi Hukuk Fakültesinin 50 Yil Armaganì (Das Recht im Zeitalter der Republik - 50-Jahre-Festschrift der Juristischen Fakultät der Universität Istanbul), Istanbul 1973, S. $1 \mathrm{ff}$.

54 Dazu Ernst E. Hirsch, Verfassungsänderungen in der Türkei (Staatsverfassungen der Welt Ergänzungsband 7a), Hamburg 1973; Bassam Tibi, Die Verfassungsänderung in der Türkei und ihr gesellschaftlicher Kontext, VRÜ 1972, S. 447 ff.; Kurt Rabl, Nochmals die türkische Verfassungsreform von 1971, VRÜ 1973, S. 219 ff. 
1961, Gewerkschaftsfreiheit Art. 46 TV 1961 u.a.m.) oder in Art. 57 TV 1961 über die Parteien; die mit der zweiten Etappe der Verfassungsreform im Jahre 1973 ins Leben gerufenen und kurz danach aufgrund eines Urteils des Verfassungsgerichts wieder aufgelösten Staatssicherheitsgerichte hatten für den strafrechtlichen Schutz der unteilbaren Einheit von Staatsgebiet und Staatsvolk zu sorgen.

b) In der Verfassung von 1982 ist ein Anstieg des Gebrauchs der Floskel zu verzeichnen: Schon die Präambel enthält sie, dann die allgemeine Schrankenbestimmung des Art. 13 Abs.1 TV 1982, die mißglückte Vorschrift über den Grundrechtsmißbrauch (Art. 14 TV 1982) und zahlreiche Grundrechtsvorschriften. Sie ist in den Bestimmungen über die Parteien (Art. 68 f. TV 1982), über die Universitäten (Art. 130 TV 1982) und über Radio und Fernsehen (Art. 133 TV 1982) zu finden. Zum Schutz der unteilbaren Einheit von Staatsgebiet und Staatsvolk (und der "freiheitlich demokratischen Ordnung" und anderer die innere und äußere Sicherheit betreffender Rechtsgüter) wird wieder die Staatssicherheitsgerichtsbarkeit eingeführt (Art. 143 TV 1982). Úberall schimmert die Angst des Verfassunggebers vor Gefahren hervor, die sich gegen den nationalen Einheitsstaat richten. Die Schrankenvorschriften, insbesondere die "Mißbrauchsvorschrift" des Art. 14 TV 1982 sind es, die der strafrechtlichen Abwehr antinationalistischer Tendenzen - früher Art. 141 Abs.4 und Art. 142 Abs. 3 türk.StGB, jetzt Art. 8 ATG - die verfassungsrechtliche Legitimation vermitteln sollen.

c) Als "allgemeines Rechtsprinzip der unteilbaren Einheit von Staatsgebiet und Staatsvolk"55 spiegelt sich dieses materiale Element des Nationalismus dann konsequent auch auf Gesetzesebene in zahlreichen Vorschriften wider, sei es im Strafgesetzbuch, wo es gar mit der Sanktion der Todesstrafe verbunden wird (Art. 125 türk.StGB)56, im Antiterrorgesetz (Art. 8 ATG und andere) oder im Parteiengesetz, auf deren Grundlage all diejenigen Aktivitäten unterbunden bzw. unter Strafe gestellt werden, die durch Handeln oder im Wege der individuellen oder kollektiven Artikulation von Gedanken die Einheit des türkischen Staates und seines Volkes in Frage stellen könnten.

d) Der Grundsatz der unteilbaren Einheit von Staatsgebiet und Staatsvolk ist für den zentralistischen Einheitsstaat Türkei so wichtig wie für die Bundesrepublik Deutschland das Föderalismusprinzip. Ersterer ist aber auch genauso unbestimmt wie der zweitere. Dies wird insbesondere dann virulent, wenn es darum geht, den durch dieses Prinzip begrenzten Schutzbereich von Grundrechten festzustellen bzw. zu konkretisieren. Der Begriff zielt

Vgl. zu dieser in ihrer Ausgestaltung durch die Rechtsprechung außerordentlich gefährlich gewordenen Vorschrift Christian Rumpf, Die asylrechuliche Relevanz der Art. 125 ff. des türkischen Strafgesetzbuches, Inf AuslR 1986, S. 250 ff.; A. Pulat Gözübüyük, Türk Ceza Kanunu (Türkisches StGB [Kommentar]), 5. Aufl., Istanbul 1990, Bd. II, zu Art. 125. 
nämlich schon seinem Wortlaut nach nicht nur auf die Unteilbarkeit des Staatsgebiets ab, sondern auch auf die Unteilbarkeit des Volkes. Mit Dönmezer ist dann zu fragen57, ob dieser Grundsatz schon dann berührt ist, wenn es nicht um eine Abtrennung von Staatsgebiet geht, sondern lediglich um kulturelle Autonomie, die etwa die Ermöglichung des Unterrichts in einer anderen Muttersprache als dem Türkischen in den Schulen einer bestimmten Region umfaßt. Man könnte auch - mit demselben Autor - noch weiter gehen und fragen, ob das Prinzip berührt ist, wenn unterschiedlichen sozialen Gruppen Sonderrechte oder Selbstverwaltungsrechte eingeräumt werden.

Zunächst darf als feststehend angesehen werden, daß der Grundsatz die Einheit der Ausübung souveräner Herrschaftsgewalt enthälth, und zwar sowohl nach außen im Sinne völkerrechtlicher Souveränität als auch nach innen im Sinne der Ausübung von Souveränität im Namen der einen Nation. Soweit es dann um die "Einheit des Staatsvolkes" geht, ist die Einheit der Nation gemeint. Für die Einheit der Nation wiederum setzt Dönmezer soziologisch an, indem er zwar die Existenz zahlreicher sozialer Gruppen akzeptiert, die jeweils ihren legitimen Interessen nachgehen, aber zugleich auf den Faktor "Integration" abstellt, ohne den es zur "Einheit" des Staatsvolkes nicht kommen kann58. Schwierig bleibt allerdings, den durch den Grundsatz der unteilbaren Einheit von Staatsgebiet und Staatsvolk geschützten Grad der Einheit festzustellen. Dönmezer - und damit dürfte er das von der Verfassung vorausgesetzte Verständnis treffen - setzt diesen Grad im Ergebnis recht hoch an: Er lehnt solche Faktoren der Abgrenzung sozialer Gruppen ab, die einen negativen Einfluß auf das Bewußtsein der Einheit bzw. das nationale Bewußtsein und damit auf die Integration der in der Nation vereinten Gesellschaft haben. Hierunter zählt er die Abgrenzung durch Sprache, Rasse, Religion, sofern diesen Elementen subjektiv die Funktion der Ausgrenzung aus der Nation zugeschrieben wird.

e) Insgesamt verhilft die Aufnahme des Grundsatzes der unteilbaren Einheit von Staatsgebiet und Staatsvolk in die Schrankenbestimmungen der Grundrechtsvorschriften und sein durch weitere Hervorhebung betonter Charakter als allgemeines Rechtsprinzip dem Nationalismus-Prinzip zu einer strukturellen Festigung, die sich in der durch den Gesetzgeber geförderten Praxis - als Beispiel sei das Parteienrecht genannt oder das Verbot der separatistischen Propaganda in Art. 8 ATG - als Antipode gegenüber dem Prinzip der freiheitlich demokratischen Ordnung auswirkt.

57 Dönmezer, S. 11 ff.

58 Dönmezer, S. 15 ff. 


\section{Die nationale Kultur}

Als weiterer integrierender Faktor verfassungspolitische und sogar verfassungsrechtliche Bedeutung gewonnen hat der Begriff der "nationalen Kultur". Er taucht erstmals in der Verfassung von 1982 auf. So will die Präambel die Grundrechtsausübung nur im Rahmen der "nationalen Kultur" erlauben; die Hohe Atatürk-Gesellschaft für Kultur, Sprache und Geschichte ist durch Art. 134 TV 1982 eigens eingerichtet worden, näher zu bestimmen, was nationale Kultur ist. Eben diese Vorschrift legt nahe, da $\beta$ die Verfassung eine nationale Kultur im Sinne des kemalistischen Nationalismus postuliert59. Wenn auch grundsätzlich ein solcher Begriff die kulturelle Vielfalt innerhalb einer Staatsnation nicht ausschließt und durchaus als Gesamtheit der "anatolischen Zivilisationen" und Kulturen - einschließlich der kurdischen - angesehen werden könnte60, so sind die Bemühungen des türkischen Erziehungsministeriums, des wichtigen Instituts für die Erforschung der türkischen Kultur und der Hohen Atatürk-Gesellschaft nicht zu übersehen, ihren Blick über die Osmanen zurück zu den türkischen Horden und Stadtstaaten Zentralasiens zu wenden, also eben doch der gegenwärtigen Wirklichkeit widersprechende ethnische Faktoren zur Geltung zu bringen. Als Beispiel seien hier nur einige of fizielle geförderte Publikationen genannt, die gerade die Kurden zum Gegenstand haben und deren türkische Abstammung nachzuweisen suchen61.

Damit bef inden sich derartige Bemühungen, die sich auch in der auf die ethnisch-türkische Kultur als nationale Kultur konzentrierten türkischen Schulhistoriographie widerspiegeln, durchaus im Einklang mit der gegenwärtigen Verfassung.

59 Der gleiche Begriff wird auch von den Vertretem der Türkisch-Islamischen Synthese verwendet (vgl. dazu Christian Rumpf, Laizismus und Religionsfreiheit, Stiftung Wissenschaft und Politik, Ebenhausen 1987, S. 97 f.). Diese einflußreiche Gruppe mit zahlreichen Vertretem in den verschiedenen Eliten (akademische, gesellschaftliche, Staatselite etc.) vertritt eine auf einem spezifischen türkischen Islam beruhende Ideologie.

60 Dafür Ekrem Akurgal, Kürtlerin Kökeni ve Türkiye'nin Kültür Bütünlügü (Die Wurzeln der Kurden und die kulturelle Einheit der Türkei), Cumhuriyet v. 8.6.1988, S. 2.

61 Sükrü M. Sekban, Kürt Sorunu (Die Kurdenfrage), 4. Aufl., Ankara 1981 (hrsg. v. Institut zur Erforschung der türkischen Kultur zum 100. Todestag Mustafa Kemal Atatürks). Die erste Auflage dieses Werks stammt aus dem Jahre 1933 (La Question Kurde, Paris). Außerdem: Nazmi Sevgen, Dogu ve Güneydogu Anadolu'da Türk Beylikleri (Die türkischen Landherrschaften in Ost- und Südostanatolien), hrsg. v. Institut zur Erforschung der türkischen Kultur zurn 100. Todestag Mustafa Kemal Atatürks, Ankara 1982; M. Serif Firat, Dogu Illeri ve Varto Tarihi (Die Ostprovinzen und die Geschichte Urartus), hrsg. v. Institut zur Erforschung der türkischen Kultur zum 100. Todestag Mustafa Kemal Atatürks, 4. Aufl., Ankara 1981 (die erste Auflage erschien 1948 in Istanbul); Sükrü K. Seferoglu, Anadolu'nun Ilk Türk Sakinleri Kürtler (Die Kurden als erste türkische Bewohner Anatoliens), hrsg. v. Institut zur Erforschung der türkischen Kultur zum 100. Todestag Mustafa Kemal Atatürks, Ankara 1982. 
Zwar enthält die Verfassung auf den ersten Blick keine eindeutige Definition der "nationalen Kultur" als ethnisch-türkische Kultur. Dennoch gibt es Hinweise auf eine derartige enge Auslegung des Begriffs. Wenn nämlich die Präambel fordert, daß keine Ansicht oder Meinung geschützt werden dürfe, die gegen die geschichtlichen und geistigen Werte des Türkentums verstößt, bringt sie das wichtigste Ausdrucksmittel von Kultur (die Rede) und den inhaltlichen Kern einer Kultur (geschichtliche und geistige Werte) in engsten Zusammenhang. Dabei liefert die Präambel zugleich einen Hinweis darauf, daß es um die ethnischtürkische Kultur zu gehen scheint. Denn anders ist der Begriff "Türkentum" (türk.: türklük) kaum zu verstehen. Und wenn, wie bereits ausgeführt, die Präambel zumindest zur Auslegung der übrigen Verfassungsvorschriften heranzuziehen ist, haben wir hier den Weg zur Auslegung des Begriffs "nationale Kultur", wie er in den übrigen Verfassungsvorschriften und - falls dort überhaupt Zweifel bestanden - auf Gesetzesebene gebraucht wird.

Bis heute gibt es Hinweise auf eine Politik der nationalen Kultur, die auf die Assimilation "konkurrierender" Kulturelemente abzielt. Ein eklatantes, aber in dieser Form inzwischen überholtes Beispiel bot das Siedlungsgesetz aus dem Jahre 193462. Dieses Gesetz formuliert in seinem Art. 1 als Ziel die Korrektur der Bevölkerungsstruktur zum Zwecke der Verbreitung der türkischen Kultur. Mittel hierzu ist die Umsiedlung von Personen, die nicht der türkischen Kultur verbunden sind, in Gegenden, in denen türkische Kultur besonders stark verbreitet ist. Dabei war gemäß dem 1947 abgeschafften Art. 11 zu beachten, daß die Gruppen klein blieben und sich nicht ganze Dörfer oder Stadtteile von Personen bilden konnten, die als Muttersprache nicht türkisch sprechen; auch die Arbeitsbeschaffung durch solche Personen für solche Personen war untersagt worden. Dieses zu Lebzeiten Atatürks in Kraft getretene Gesetz zum Zweck der endgültigen Lösung der Kurden- bzw. Nationalitätenfrage ist nicht konsequent, sondern nur wellenweise umgesetzt worden63. Heute ist sein Anwendungsbereich auf Einwanderer, Flüchtlinge und Nomaden begrenzt.

In einem ethnozentrischen Sinne läßt sich auch Art. 81 des Parteiengesetzes verstehen64, wonach politische Parteien nicht behaupten dürfen, daß es auf unterschiedlicher Sprache oder Rasse beruhende Minderheiten gebe, und keine andere Kultur oder Sprache fördern

62 Gesetz Nr. 2510 v. 14.6.1934 (RG Nr. 2733 v. 21.6.1934) mit späteren Änderungen.

63 Umfangreiche Umsiedlungsaktionen der letzten Jahre dürften allerdings weniger auf das Siedlungsgesetz gestützt worden sein als auf die Notstandsgesetzgebung und das Waldgesetz (vgl. die Presseberichte in Yüzyil v. 23.9.1990; 2000e Dogru v. 28.1.1987, 7.1.1990, 28.1.1990 und 20.5.1990; Cumhuriyet v. 17.2.1987, 18.2.1987, 20.2.1987, 13.11.1989 und 22.12.1989 [zu einem Bericht des Menschenrechtsvereins Diyarbakìr]). Dennoch weisen der Umfang der Aktionen (viele Hunderte von Dörfem) und die Entfemungen (vom Osten bis an die Küsten der Ägäis) darauf hin, daß eine Entwurzelung der betroffenen Bevölkerungen und damit eine Assimilation in die "türkische Kultur" Westanatoliens gefördert werden soll.

64 Abgedruckt bei Ernst E. Hirsch, Die Verfassung der Türkischen Republik, JöR 32 (1983), S. 507 ff. (S. 607). 
oder vertreten dürfen als die türkische Kultur oder Sprache. Auch hier zeigt sich wieder eine Bevorzugung der ethnisch-türkischen Kultur. Zwar könnte man unter "türkische Kultur" hier durchaus noch eine nationale Kultur aller türkischen Staatsangehörigen erkennen, was dann sogar das Verbot der Pflege einer ethnisch-türkischen Kultur durch die politischen Parteien zur Folge hätte. Doch bedürfte eine solche Auffassung angesichts der unmittelbaren Verknüpfung zwischen dem Begriff der türkischen Kultur und dem tragenden Element, nämlich der türkischen Sprache, eines großen Überzeugungsaufwandes. Hinzu tritt die im gleichen Zusammenhang ausgesprochene Ausgrenzung "anderer" Kulturen. Die neue Praxis des Verfassungsgerichts hat hier Schwierigkeiten, dem aus der Abgrenzung von "türkischer" gegenüber "kurdischer" Kultur erwachsenden alten Mißverständnis entgegenzutreten, dies bedeute die Gleichstellung von nationaler Kultur und ethnisch-türkischer Kultur65.

Es ist liegt also nahe anzunehmen, daß das Rechtsprinzip der nationalen Kultur der türkischen Verfassung sich auf einen ethnisch-türkischen Kulturbegriff als ethnisch-türkische Kultur stützt und damit der Geltendmachung anderer ethnischer Kulturelemente entgegenwirkt.

\section{Das Mißverständnis des Gleichheitssatzes}

Art. 69 TV 1924 war bei der Formulierung des Gleichheitssatzes noch ohne die Verwendung des Begriffs "Rasse" ausgekommen und hatte auf die Aufhebung von Gruppen-, Klassen- und Familienprivilegien abgezielt. Art. 12 TV 1961 und dann auch Art. 10 TV 1982 haben den Gleichheitssatz entsprechend den völkerrechtlichen Regeln zum Schutz der Menschenrechte weiter nach Sprache, Rasse, Geschlecht, politischer Meinung, Weltanschauung, Religion und Bekenntnis ausdifferenziert, die nicht zum Maßstab unterschiedlicher Behandlung gemacht werden dürfen.

Die türkische Praxis hat daraus etwas Ungewöhnliches gemacht: Der Spieß wurde gegen diejenigen umgedreht, die sich auf ihr Anderssein, auf Regeln des Minderheitenschutzes berufen wollten. Der Gleichheitssatz, der eigentlich dem Schutz des gleich zu behandelnden Individuums gegen den Staat dient, wird für eine Ermächtigung zugunsten des Staates gehalten, die "Schaffung einer Rasse oder einer ethnischen Gruppe" zu unterbinden, objektiv gegebene und von den Grundrechtsträgern zudem selbst gewollte (und durch das moderne Völkerrecht geförderte) Ungleichheiten in ethnischer und religiöser Hinsicht

durch Assimilation zu verhindern oder einzuebnen. Dadurch verliert der Gleichheitssatz seine grundrechtebezogene Schutzfunktion und wird zur Waffe des Staates gegen das Indi- 
viduum denaturiert. Es wird der im Gleichheitssatz enthaltene Anspruch auf Ungleichbehandlung verkannt, sofern es eine sachliche Rechtfertigung gibt.

Diese eigenartige Auffassung führt zu einer Fiktion nicht nur der rechtlichen, sondern auch der sachlichen Gleichheit aller türkischen Staatsbürger. Damit die Norm paßt, wird ihr Sachbereich, der ihr zugrundeliegende Lebenssachverhalt manipuliert. Dieser Fiktion gemäß gab es keine Kurden, wer sich als Kurde bezeichnete bzw. die Existenz des kurdischen Volkes behauptete, schuf eine ethnische Gruppe. Dies gilt - abgeschwächt durch die neue reale Kurdenpolitik - noch heute.

\section{Die Staatsangehörigkeit}

Wichtige Hinweise auf das Nationalismus-Verständnis vermittelt die Regelung der Staatsangehörigkeit. Art. 54 TV 1961 bzw. Art. 66 Abs.1 TV 1982 lauten:

"Jeder, den mit dem Türkischen Staat das Band der Staatsangehörigkeit verbindet, ist Türke".

Die Notwendigkeit, den "Türken" in einer Verfassungsvorschrift zu definieren, weist von selbst darauf hin, daß wir es mit einer Verfassung zu tun haben, die das Problem Kulturnation/Staatsnation in staatsnationalem Sinne zu lösen hat. Die Verfassung verzichtet hier ausdrücklich und konsequent auf eine nähere Spezifizierung des "Türken" nach ethnischen, rassischen, sprachlichen oder sonstigen Gesichtspunkten und formuliert einen staatsrechtlichen Begriff des "Türken". Geht man davon aus, daß der Verfassunggeber objektiv gewußt hat, da $B$ das türkische Staatsvolk uneinheitlicher ethnischer Herkunft ist, so kann dies nur heißen, daß der verfassungsrechtliche Nation-Begriff an die Staatsangehörigkeit anknüpft66. Es kann hieraus kein Zwang der Assimilation oder auch nur der unfreiwilligen Gleichbehandlung aller Türken geschlossen werden, soweit es um kulturelle Identitätsmerkmale geht. Als Türken gleich sind sie als Staatsbürger, nicht aber dort, wo sie faktische Unterschiede aufweisen wie zum Beispiel als Zugehörige verschiedener Volksgruppen.

"Türke" wird man also mit Annahme der türkischen Staatsangehörigkeit, sei es durch Geburt, sei es - wie in den dreißiger und vierziger Jahren bei vielen deutschen Wissenschaftlem im türkischen Exil - durch Verleihung aufgrund Ministerratsbeschlusses oder sei es aufgrund der Eheschließung mit einem türkischen Staatsbürger. Damit ergeben sich die spezifischen staatsbürgerlichen Rechte und Pflichten vom Wahlrecht über den Militärdienst bis zu den Auswirkungen im internationalen Privatrecht. Mehr bedeutet die Verfassungs-

So etwa auch Cihan (Fn. 43) S. 54. 
vorschrift nicht, so daß ethnische, soziale, rassische und religiöse Gegebenheiten unberührt bleiben. Art. 66 Abs.1 TV 1982 präkludiert also nicht das ethnische, soziale, rassische oder religiöse "Anderssein" im Rahmen des türkischen Staatsverbandes, also nicht einmal die Existenz von Minderheiten im rechtlichen Sinne67.

\section{Die Sprache}

Schon mit Art. 2 TV 1924 wurde die Amtssprache auf das Türkische festgelegt. Úbernommen wurde dies durch Art. 3 TV 1961 und, leicht modifiziert, durch Art. 3 TV 1982, wo es heißt: "Seine (i.e.: des Staates) Sprache ist Türkisch".

Nachvollziehbar ist an solchen Vorschriften, wie sie es auch in anderen Verfassungen gibt (z.B. Frankreich), die denkbare Auffassung, daß die Sprache einen Kommunikationsfaktor darstellt, der für das Funktionieren einer Staatsbürokratie von Bedeutung sein kann68. Solches gilt vielleicht noch für Vorschriften wie Art. 68 Abs.2 TV 1961, wonach Abgeordneter nur werden konnte, wer Türkisch lesen und schreiben kann69; den gleichen Effekt hat, wenn auch weniger deutlich, die entsprechende Vorschrift des Art. 76 Abs.2 TV 1982, wonach ein Abgeordneter zumindest Grundschulausbildung haben muß, die ausschließlich in türkischer Sprache erfolgt. Bedenklich und zur Ermächtigungsgrundlage für eine rücksichtslose Assimilationspolitik können dann aber Vorschriften wie Art. 26 Abs.3 S.1 TV 1982 (Verbot der Meinungsäußerung in einer durch Gesetz verbotenen Sprache) und Art. 28 Abs.2 TV 1982 (Verbot der Veröffentlichung in einer durch Gesetz verbotenen Sprache) werden, die - wie es sich dann auch 1983 in dem erst durch Art. 23 ATG im Jahre 1991 wieder aufgehobenen Sprachenverbotsgesetz realisiert hat 70 - gegen die Verwendung nichttürkischer Sprachen im politischen oder privaten Alltag zum Einsatz kommen können.

Die Sprachregelungen der türkischen Verfassung geben damit dem Nationalismus eine ethnozentrische bzw. assimilatorische Färbung. Obwohl grundsätzlich die Normierung einer "offiziellen" Sprache so wenig wie die Definition des "Türken" über die Staatsangehörigkeit dem ethnischen, muttersprachlichen und sonstigen Anderssein entgegenstehen muß, so greift die türkische Verfassung mit den Art. 26 und 28 TV 1982 nun doch auf die

67 In gegenteiliger Richtung aber interpretiert die türkische Regierung den Art. 66 Abs. 1 TV 1982, der in ihrer Begründung des Vorbehalts zu Art. 27 des UN-Paktes über die bürgerlichen und politischen Rechte auftaucht (Cumhuriyet v. 23.7.1992).

68 Dies ist, wie viele Beispiele funktionierender Staatswesen mit verschiedenen Amtssprachen zeigen (Schweiz, Belgien, Kanada, USA [Neu Mexiko u.a.], Spanien [Katalanien] etc.), nicht zwingend.

69 Wäre nur daran gedacht worden, die Wahl von Analphabeten zu verhindem, hätte es der Einführung des Worts "Türkisch" nicht bedurft.

70 Dazu ausführlich Christian Rumpf, Das türkische Sprachenverbotsgesetz unter besonderer Berücksichtigung der völkerrechtlichen Verpflichtungen der Türkei, Orient 1989, S. 413 ff. 
Muttersprache zu, auf die Sprache als privatem Kommunikationsmittel und wesentlichem Träger und Vermittler kultureller Identifikation; auch Art. 42 Abs.9 TV 1982, der allein das Türkische als "Muttersprache" zu lehren erlaubt, deutet in die gleiche Richtung, obwohl er den Unterricht in anderen Sprachen als "Fremdsprachen" - also insoweit etwa auch im Kurdischen - nicht ausschließt.

Auf Gesetzesebene ist die Sprachenregelung der Verfassung nicht nur im bereits erwähnten Sprachenverbotsgesetz aus dem Jahre 1983 umgesetzt worden, das im Jahre 1991, als die Kurdenfrage in der innertürkischen Diskussion völlig neuen Höhepunkten zusteuerte, wieder zurückgenommen wurde. Vielmehr ist diese Regelung auch in die Gesetzgebung über die Vereine, Parteien, berufsständischen Vereinigungen, zum Bildungswesen etc. übernommen und bis heute erhalten worden. Die türkische Sprache hat damit über das Monopol als Amtssprache hinaus überhaupt das Monopol in allen Bereichen gesellschaftlicher Offentlichkeit und damit den Charakter als zentraler Integrationsfaktor zugeschrieben bekommen.

\section{Die Religion}

Der Begriff der Nation selbst bildet, wie oben bereits erwähnt, einen Schnittpunkt zwischen islamischer Religion und Nationalismus.

Als "millet" spielt der Begriff der Nation im Islam und seinem Recht eine ganz wesentliche Rolle. Bekanntlich sind die Rechtsverhältnisse der Menschen - seien es die des Strafrechts, des Zivilrechts oder des Staatsrechts - im Koran vorgezeichnet, zum Teil auch durchaus präzise bestimmt. Dabei unterscheidet der Koran jedoch zwischen den Gläubigen (den Bürgern der Welt des Friedens) und den Ungläubigen (den Bürgern der Welt des Krieges). Bürger der Welt des Friedens konnten jedoch nicht nur die Muslime sein, sondern auch solche Nichtmuslime, denen durch Verträge oder auf andere Weise der Status von Schutzbefohlenen zugewiesen worden war. Dies war der Status, der bestimmte Gruppen der Nichtmuslime mit Heiligen Büchem, darunter vor allem die Juden und Christen, zu eigenen Nationen machte, die der islamischen Welt (dar ül-Islam) als einziger und umfassender Gemeinschaft der Gläubigen gegenüberstehen. Abgrenzungsmerkmal der Nationen ist also die Religion; die Religionszugehörigkeit vermittelt die Nationalität71. Territoriale Verhältnisse, die für den modernen Staatsbegriff konstitutionelle Bedeutung haben, treten dabei in den Hintergrund. Dies war auch eine der wichtigsten Grundlagen der osmanischen Verfassungsordnung.

$71 \mathrm{Da}$ sich in der Praxis schon bald nach Mohammeds Tod auch die islamische Welt konfessionell geteilt hatte und von der Entwicklung modemer Staatlichkeit nicht verschont wurde, ist hier ohne Bedeutung. 
Gezeigt werden soll, daß die türkische Verfassung trotz des ausdrücklichen Bekenntnisses zum kemalistischen Laizismus einen Weg gesucht hat, wenigstens punktuell das Integrationsdefizit eines säkularen Nationalismus in der islamischen Türkei auszugleichen, indem sie - wie Mustafa Kemal Pascha vor der Großen Nationalversammlung im Jahre 192072 daran erinnert, daß das "Band der Staatsangehörigkeit" durch die gemeinsame Religion zusätzlich gef estigt werden kann - wenn auch nur im Hinblick auf die Mehrheit der Bevölkerung.

Hinzuweisen ist hier auf Art. 136 TV 1982, der die Bestimmung über das Präsidium für Religionsangelegenheiten enthält. Dieses Präsidium, dessen Ubereinstimmung mit einem strikten Laizismus-Konzept recht fragwürdig ist, dient in erster Linie dazu, den Islam als gesellschaftlichen Faktor unter staatliche Kontrolle zu bringen. Eine kleine Floskel in jener Vorschrift weist jedoch auf einen interessanten Zusammenhang mit dem säkularen Nationalismus hin: Das Präsidium soll nämlich seine Aufgaben im "Hinblick auf nationale Solidarität und Integration" erfüllen. Damit wird - scheinbar ganz unkemalistisch - der Islam zum Intergrationsfaktor für die türkische Nation. Denn was der Laizismus nicht leistet, kann der Islam leisten: das gemeinsame Bewußtsein der überwiegend muslimischen Bevölkerung einschließlich ethnischer Minderheiten für eine gemeinsame Identität fruchtbar machen.

Dem dahintersteckenden, letztlich aus der osmanischen Staatsverfassung des 19. Jahrhunderts tradierten Gedankengut entspricht auch die offizielle These, daß es in der Türkei keinerlei Minderheiten gebe - vorbehaltlich derer, die ihren Status durch den Lausanner Vertrag von 1923 erhalten hätten. Das aber sind nur bestimmte christliche Gemeinschaften und die Juden - die alten islamischen "millets".

Grundsätzlich wird man diese Regelung durchaus als mit einem pragmatischen Kemalismus vereinbar ansehen können, zumal ja das Präsidium für Religionsangelegenheiten, wenn auch ohne Verfassungsrang, schon zu Atatürks Zeiten existiert hatte. Problematisch ist allein - neben der Erhebung in den Verfassungsrang - der Umstand, daß der Islam als Integrationsfaktor eben nur die Mehrheit der türkischen Bevölkerung erfassen kann. Die Folge ist wieder unvermeidlich eine stärkere Betonung kultureller Elemente des Nationbegriffs und damit eine Ausgrenzung solcher türkischer Staatsangehöriger im Sinne des Art. 66 Abs.1 TV 1982 als "Nicht-Türken", denen dieses integrative Element der gemeinsamen Religion fehlt - seien es Angehörige von durch den Lausanner Vertrag geschützten und privilegierten Minderheiten oder seien es Angehörige von Minderheiten, die noch nicht 
einmal dem Regime des Lausanner Vertrages unterstehen und damit in ihrem kulturellen Anderssein völlig ungeschützt sind 73 .

\section{Der zentralistische Einheitsstaat}

Wenn bisher schon erkannt worden ist, wie sehr die türkische Verfassung der Einheit von Staatsgebiet und Staatsvolk zu dienen bestimmt ist, so findet dies seinen Ausdruck auch in der bürokratischen Struktur der Republik als zentralistischer Einheitsstaat. An der Spitze steht als einzige Gesetzgebungskörperschaft die Große Nationalversammlung der Türkei als integrativer Repräsentant der Volkssouveränität und der Souveränität der Nation. Dezentrale Elemente sind allein im Bereich der Exekutive auszumachen, dort vor allem in verwaltungstechnischer Hinsicht. Zwar sehen Art. 115 Abs.3 TV 1961 und heute Art. 126 Abs.3 TV 1982 "Regionen" vor, sie dienen jedoch lediglich der ökonomischeren Befriedigung einzeln definierbarer Bedürfnisse (z.B. Kultur, Verteidigung, Wirtschaft u.a.) mit dem Ziel eines gesamtstaatlichen Gleichgewichts. Der 1981 gemachte Ansatz zur Schaffung fester Regionen mit eigenen Verwaltungskompetenzen nach französischem Vorbild blieb in der Verabschiedung einer Rechtsverordnung mit Gesetzeskraft stecken, die nie umgesetzt und später vom Parlament zurückgenommen wurde. Allein der Regionalpräfekt der Notstandsverwaltung läßt sich als begrenzte Wiedergeburt jenes Ansatzes erkennen - aber eben nur für den Notstand74. Regionalismus und Föderalismus gelten darüberhinaus nach wie vor als mit dem Grundsatz des zentralistischen Einheitsstaates nicht vereinbar, ja werden wie anläßlich der Rücknahme der genannten Rechtsverordnung mit Gesetzeskraft behauptet - als "Separatismus" diskreditiert. Der Umstand, daß die gegenwärtige Regierung unter Süleyman Demirel eine föderale Lösung der Kurdenfrage ablehnt, weist auf einen engen Zusammenhang zwischen Nation-Verständnis und bürokratischer Struktur hin. Das Bestehen auf dem Zentralismus erweist sich nur als sinnvoll, wenn er dazu dient, die Einheit einer Staatsnation zu erhalten. Ein so enges Nationalismus-Verständnis geht von einer Einheit von Staatsgebiet und Staatsvolk aus, die sich im Sinne des Staatsnationsbegriffs vor allem an den äußeren, völkerrechtlichen Grenzen des Staates orientiert und über die praktische Unterteilung in rein exekutive Verwaltungseinheiten hinausgehende Gewährung auch nur partieller, allein in Relation zu zentralen Staatsgewalt stehender Souveränitätsrechte nicht zuläßt.

$73 \mathrm{Zu}$ den aufgrund des Lausanner Vertrages nicht "privilegierten" Minderheiten gehören unter anderem die Suryanî (syrisch-orhodoxen Christen, auch als aramäische Christen bezeichnet) und die Yezidî (irreführend auch als Teufelsanbeter bekannt).

74 Vgl. hierzu Christian Rumpf, Der Not- und Ausnahmezustand im türkischen Verfassungsrecht, ZaöRV 48 (1988), S. 683 ff. 


\section{Die Auffassung des türkischen Verfassungsgerichts}

Eine deutlich weniger ethnozentrische Variante des Nationalismus vertritt das türkische Verfassungsgericht. Exemplarisch hierfür ist sein Urteil vom 16.7.1991, mit dem die Vereinigte Türkische Kommunistische Partei verboten wurde75. Grund des Verbots waren nicht etwa kommunistische Elemente im Parteiprogramm. Denn insoweit wurde die Partei angesichts der zeitgeschichtlichen Entwicklungen in den "Eurokommunismus" eingeordnet, der nicht mehr die Merkmale des klassischen Kommunismus im Sinne des orthodoxen Marxismus-Leninismus aufweise. Vielmehr rügte das Verfassungsgericht die programmatische Zuwendung der Partei zum Schutz kurdischen Volkstums76.

Die Verfahrensvertreter der Partei hatten einen "weichen", multikulturellen Nationalismus unter dem Schlagwort "Einheit in der Vielfalt" vertreten. Dies wurde vom Verfassungsgericht unter Berufung auf einschlägiges Verfassungs- und Gesetzesrecht abgelehnt.

Die oben 5. zitierte Vorschrift des Art. 81 des Parteiengesetzes legte das Verfassungsgericht im Lichte der Vorschrift über die Staatsangehörigkeit aus. Bei dem Begriff "Türkisch" handle es sich nicht um einen ethnologischen, sondern um einen reinen Rechtsbegriff, der über das Band der Staatsangehörigkeit definiert sei. Damit kam es zu einem hieran ausgerichteten Begriff der nationalen Kultur. Es bestritt den Kurden ausdrücklich keineswegs sprachliche und kulturelle Eigenheiten. Es sei jedoch einer Partei versagt, diese Eigenheiten politisch zu bewerten und gegenüber der nationalen Kultur abzugrenzen.

Das Verfassungsgericht stützte dieses Ergebnis auf eine ausführliche Bewertung des Rechtsprinzips der unteilbaren Einheit von Staatsgebiet und Staatsvolk, die verfassungsrechtlichen Regelungen zur Sprache und schließlich auf die Ablehnung der Möglichkeit der Existenz von Minderheiten, soweit nicht ausnahmsweise durch den Lausanner Vertrag zugelassen.

Die Ausscheidung der Existenz eines Rechtsbegriffs der Minderheit wird vom Verfassungsgericht über eine Definition des Begriffs der Nation als Staatsnation vorgenommen. Es hält die Nation für die am höchsten entwickelte soziale Gemeinschaft der Menschen, die sich von jeder Abstammungsgemeinschaft oder Rasse abhebt. Es rechnet einen auf ethnische Merkmale gestützten Nation-Begriff den primitiveren Gemeinschaftsformen zu77.

Anschließend formuliert das Verfassungsgericht eine pragmatisch-aufgeklärte Variante des kemalistischen Nationalismus. Das Verfassungsgericht verweist auf die Geschichte der

75 Fn. 65.

76 Verf, a.a.O., S. $71 \mathrm{ff}$.

77 A.a.O., S. 78. 
letzten Jahrtausende auf dem Boden Anatoliens, die zu einer Vielfalt der Völkerschaften geführt habe. Über den "nationalen Pakt" (Misakì Millî), mit dem zu Beginn des Unabhängigkeitskrieges die Grenzen der neuen Türkei festgelegt worden waren78, sei der große auf gemeinsamem Recht, gemeinsamer Tradition und Geschichte, gemeinsamer Moral und Gleichberechtigung beruhende Konsens des neuen Staatsvolkes der modernen Türkei hergestellt worden. Wenn auch verschiedene Ethnien auf dem Boden der Türkei verschiedene kulturelle Merkmale auf wiesen, so seien diese jedoch in einer Gesamtkultur verschmolzen worden, die nunmehr der türkischen Nation eigen sei. Welcher Wurzel auch die einzelnen Gruppen seien, eine Unterscheidung nach ethnischen, religiösen und ähnlichen Merkmalen sei rechtlich nunmehr ausgeschlossen. Dies drücke das Rechtsprinzip der unteilbaren Einheit von Staatsgebiet und Staatsvolk aus. Genauso sei auch jede andere Form des Nationalismus wie Turanismus, religiöser Nationalismus und ein sonstiger an rassische Merkmale anknüpfender Nationalismus abzulehnen79.

Im Ergebnis vertritt also das türkische Verfassungsgericht einen rationalen, antirassistischen Nation-Begriff und Nationalismus, der auf der rechtlichen Negation ethnischer Abgrenzungsmerkmale beruht.

\section{Das Spannungsfeld zwischen Nationalismus, Demokratie und Grundrechten}

Der Text der türkischen Verfassung selbst legt eine Untersuchung des Spannungsfeldes nahe, das zwischen Demokratie und Grundrechten einerseits und Nationalismus andererseits besteht. Anders als der Grundgesetzgeber für die Bundesrepublik Deutschland hat der türkische Verfassunggeber ein Bedürfnis empfunden, den Nationalismus bzw. seine Elemente als eigene Rechtsgüter zu formulieren und in verschiedene Vorschriften sowohl im Grundrechteteil als auch im Staatsorganisationsteil aufzunehmen. Dies äußert sich positivrechtlich in der Formulierung entsprechender Grundrechtsschranken und in diesem Zusammenhang in einem besonderen Verhältnis zum Demokratieprinzip, das sich dann weniger als ergänzendes denn als konkurrierendes Prinzip darstellt.

78 Diese Grenzen stimmen im Osten nicht ganz mit der heutigen Grenze überein.

79 Vgl. auch VerfG, Urt. v. 28.11.1980, E.1979/31, K.1980/59, AMKD 18, S. 339 ff. (Verfassungsmäßigkeit von Art. 142 türk.StGB). 


\section{Der Nationalismus in den Grundrechtsschranken}

Als ausdrückliche Grundrechtsschranken tauchen verschiedentlich die nationale Sicherheit 80 und die unteilbare Einheit von Staatsgebiet und Staatsvolk auf (Art. 13 Abs.1 [allgemeine Schranken]; Art. 28 Abs.5 und 7 [Pressefreiheit]; Art. 30 Abs.1 [Schutz der Pressemittel], Art. 33 Abs.6 [Vereinsfreiheit], Art. 34 Abs.4 TV 1982 [Demonstrationsrecht]). Sie begrenzen den Schutzbereich der betreffenden Grundrechtsnormen zum Teil erheblich und geben die Ermächtigungsgrundlage für die gegenwärtige Gesetzeslage her 81 . Damit erhält der Nationalismus bedeutenden Einfluß nicht nur auf die Staatsorganisationsverfassung, sondern auch auf das Grundrechteregime. Es entsteht der Eindruck, da $B$ nicht mehr der Bürger, das Individuum im Mittelpunkt des Grundrechteteils steht, sondem bestimmte Elemente des öffentlichen Interesses, Nation und Staat.

Dieses negative Ergebnis kann verfassungsdogmatisch relativiert werden; dem türkischen Verfassungsgericht fällt hier eine Aufgabe zu, der es noch zögernd nachkommt, wie das oben ausführlich zitierte Urteil zeigt.

\section{Die Schranken des Nationalismus-Prinzips (Schrankenschranken)}

Das verfassungsdogmatische Instrumentarium zu besagter Relativierung ergibt sich vor allem aus Art. 13 TV 1982. Er enthält nämlich nicht nur die allgemeinen Schranken, sondern auch eine "Schrankenschranke": sämtliche Schranken sämtlicher Grundrechte sind ihrerseits begrenzt durch die "Erfordernisse einer demokratischen Gesellschaftsordnung". Diese Schrankenschranke ist zu Recht in letzter Zeit vom Verfassungsgericht schon öfters fruchtbar gemacht worden, wenn es um durch die primären Schranken scheinbar gerechtfertigte Einschnitte in Grundrechtsgewährleistungen ging82. Als weitere - immanente Schrankenschranke wurde auch das Rechtsstaatsprinzip als Quelle des Verhältnismäßigkeitsgrundsatzes herangezogen, um die Durchsetzung von Grundrechtsgewährleistungen gegen scheinbar primär gerechtfertigte Beschränkungen sicherzustellen 83 und somit dem

80 Vgl. z.B. Tayfun Akgüner, 1961 Anayasasìna Göre Milli Güvenlik Kavramì ve Milli Güvenlik Kurulu (Der Begriff der Nationalen Sicherheit und der Nationale Sicherheitsrat gemäß der Verfassung von 1961), Ankara 1983.

81 Gemäß Übergangs-Artikel 15 TV 1982 kann die gegenwärtige Gesetzeslage noch in verfassungswidriger, aber unanfechtbarer Weise darüber hinausgehen, soweit es sich um Gesetze handelt, die während der Übergangszeit unter dem Militärregime 1980 bis 1983 erlassen worden sind. Gegen sie darf nämlich "nicht die Behauptung ihrer Verfassungswidrigkeit" aufgestellt werden (vgl. Fn. 52).

82 Urt. v. 26.11 .1986$, E.1985/8, K.1986/27, in: RG 19544 v. 14.8.1987, S. 13 ff. (teilweise Nichtigerklärung eines Änderungsgesetzes zum Polizeigesetz).

83 Urt. v. 1.10.1991, E.1990/40, K.1991/33, in: RG Nr. 21135 v. 7.2 .1992 (Nichtigerklärung der Einführung eines rechtswegbeschränkenden "Beschwerdewegs"). 
Demokratieprinzip zur Durchsetzung zu verhelfen. Dies bedeutet zugleich, daß die einer modernen Demokratie84 eigenen Grundrechte in ihrem Wesensgehalt nicht angetastet werden dürfen, vielleicht könnte sich sogar eine Wendung von der Neigung der Verfassungsinterpreten, sich in dubio pro auctoriate zu entscheiden, hin zum Grundsatz in dubio pro libertate ergeben. In diesem Sinne wird also das Verfassungsgericht seine Rechtsprechung noch liberalisieren können, vielleicht sogar soweit, wie es die Verteidigung im Verfahren des Verbots der Vereinigten Kommunistischen Partei gefordert hatte, nämlich im Sinne eines Nationalismus-Prinzips, das vom Grundsatz der Einheit in der Vielfalt beherrscht ist 85 .

\section{Sonderfall Sprachverbot}

Etwas schwieriger gestaltet sich jedoch eine Öfnung des Nationalismus-Prinzips hinsichtlich der Sprache. Denn dem Gesetzgeber ist es ohne weiteres erlaubt worden, den Gebrauch einer Sprache gesetzlich zu verbieten. Die entsprechenden Vorschriften, nämlich Art. 26 Abs.3 S.1 und Art. 28 Abs.2 TV 1982, sind zwar ebenfalls im Lichte der allgemeinen Schranken und der Schrankenschranken des Art. 13 TV 1982 auszulegen; die Folge hiervon ist, daß der Gebrauch einer Sprache jedenfalls dann nicht untersagt werden kann, wenn dies dazu führt, daß einem Teil der Bevölkenung das Sprechen schlechthin verboten würde. Andererseits muß aber auch in Betracht gezogen werden, daß der Verfassunggeber mit dieser extremen Sanktion dem Gesetzgeber gerade auch ein Mittel an die Hand geben wollte, das Erlernen und den Gebrauch der Nationalsprache "Türkisch" zu erzwingen. Andemfalls liefe diese Verfassungsvorschrift völlig leer. Hinsichtlich der Möglichkeit des Sprachverbots scheint also die Durchsetzung des Demokratieprinzips gegen das insoweit rigide Nationalismus-Prinzip problematisch, so da $\beta$ hier jedenfalls der verfassungändernde Gesetzgeber eingreifen sollte.

84 Das Verfassungsgericht hat festgestellt, daß der Demokratie-Begriff der türkischen Verfassung kein spezifisch türkischer, sondem ein universaler Begriff ist, so daß auf die wesentlichen Merkmale einer europäischen Demokratie zurückgegriffen werden kann (vgl. VerfG Urt., a.a.O., [Fn. 83]). Wichtige Maßstäbe vermittelt daher also zum Beispiel die Praxis der Organe der Europäischen Menschenrechtskonvention, deren Rechtsprechung die türkische Regierung anerkannt hat.

85 Angesichts der rigiden Fassung des Art. 81 des Parteiengesetzes, das wegen der bedauerlichen Fortgeltung des Úbergangs-Art. 15 TV 1982 (dazu das VerfG [Fn. 52 und 65]) nicht für verfassungswidrig erklärt werden kann, sondern der Änderung durch den Gesetzgeber harr, ist insofem eine Änderung der Parteienrechtsprechung nicht möglich. 


\section{Schluß}

Der ideologische Gehalt der türkischen Verfassung kommt vor allem im NationalismusPrinzip zum Ausdruck, wobei sie gegenwärtig eine ethnozentrische Interpretation dieses Prinzips erlaubt. Verantwortlich für eine in diesem Sinne enge Interpretation des Nationalismus in der türkischen Verfassung sind die Elemente bzw. Rechtsprinzipien, die den türkischen Nationalismus-Gedanken in der von der Verfassung vorgenommenen Ausformung prägen, nämlich die unteilbare Einheit von Staatsgebiet und Staatsvolk und die nationale Kultur - letztere ausgestattet mit der extremen Sanktionsmöglichkeit des generellen Verbots einer anderen Sprache als des Türkischen im behördlichen oder alltäglichen Gebrauch und als ethnisch-türkische Kultur gedacht. Vor allem von diesen beiden Prinzipien geht aufgrund positiven Verfassungsrechts ein assimilatorischer Druck aus, der Begriffe wie die der "Nationalität" oder "Minderheit" bis auf wenige Ausnahmen (Lausanner Vertrag) ausschließt.

Der Nationalismus der türkischen Verfassung beruht nicht nur auf einer Abgrenzung nach außen als Grundlage völkerrechtlicher Souveränität, sondern zielt auch auf die Einheit der Souveränitätsausübung nach innen ab, auf die Integration des Staatsvolkes in einer Staatsnation. Integration bedeutet zugleich Assimilation bzw. Verschmelzung aller kulturellen Elemente zu einer nationalen Kultur. Im Extremfall führt dies zu der eben erwähnten Gleichstellung von nationaler Kultur und ethnisch-türkischer Kultur, in jedem Falle aber wird die Möglickeit politischer Abgrenzung zwischen verschiedenen ethnischen Gruppen und das Postulat der Existenz von Minderheiten im Rechtssinne versagt, gegebenenfalls zulasten eines rationalen Gleichheitsverständnisses und der Ausübung von Grundrechten, die die Zugehörigkeit zu einer Minderheit manifestieren könnte. Der Nationalismus gerät damit zur Antipode nicht nur eines Regionalisierungs- oder Föderalismusgedankens, sondern auch des Demokratieprinzips schlechthin.

Ansätze zur Aufbrechung einer derart rigiden, unitarisch-nationalistischen Verfassungsordnung sind jedoch nicht völlig ausgeschlossen. Denn sowohl Gesetzgeber als auch Verfassungsgericht und Justiz haben die Möglichkeit, durch moderne Auslegungstechniken wie die Herstellung praktischer Konkordanz zwischen "Nationalismusprinzip" und "Demokratieprinzip" und konsequente Anwendung rechtsstaatlicher Interpretationsmaßstäbe wie "Verhältnismäßigkeitsprinzip" oder "in dubio pro libertate" eine Anpassung der Verfassungsordnung an europäische Standards herbeizuführen. So hat das Verfassungsgericht immerhin versucht, eine moderne Variante des kemalistischen Nationalismus zu vertreten, die ethnozentrische Tendenzen ablehnt. Wenn es auch nicht konkurrierende Kulturen neben der "nationalen Kultur" auf dem Boden der Republik Türkei dulden mag, so definiert es doch diese nationale Kultur entsprechend den ethnischen Gegebenheiten und der Geschichte auf türkischem Boden in einer Weise, die ein Monopol der ethnischen Türken nicht kennt und damit zugleich andersartige kulturelle Elemente anerkennt. 
Hier liegt ein noch ungenügend genutzter Ansatzpunkt für eine Verfassungsentwicklung, die die durch einen zu rigiden Nationalismus gehemmte Anpassung der türkischen Verfassung an europäische Standards ermöglicht. 


\section{The Principle of Nationalism in the Turkish Constitution}

\section{By Christian Rumpf}

Turkey is a national State, and not a State of nationalities. On the background of the Kurdish question which has become one of the most existential problems of the Republic of Turkey during the last years and which has been opened to public discussion about two years ago, it seems to be interesting to find out how far the Turkish Constitution is prepared for considerable changes in minority policies. As a matter of fact, the normative structure and contents of the Constitution is ruled by the principles of Kemalism as the ideology of the State, first of all by the principle of nationalism. This article tries to explain of Kemalism bears - in the Turkish theory and practice - the characteristical elements of an ideology, even if in our opinion Kemalism should be considered as a "Weltanschauung" with utmost flexibility towards any democratic policy following Western European patterns. As "revolutionary reformism" is one of the "six arrows" - the others are republicanism, secularism, nationalism, populism and etatism -, Kemalism is open to any development according to national and international exigencies as long as the unity of the State and its nation is guaranteed.

However, most Turkish scholars and even jurisprudence do not seriously attempt to change their view on Kemalism being an ideology. As a consequence, Kemalism turns out comprising all elements of what renders an ideology comparable to religion, with its sacred symbols, faith and services.

As such, Kemalism found its way into the Constitution, today even more than in the Constitution of 1961 or the Constitution of 1924, into which the "six arrows" had been incorporated only in 1937. Thus, the Turkish Constitution obtained an extremely ideological character, in some respect incompatible with the exigencies of the principle of democracy. Out of the "six arrows" mentioned above, nationalism is the one which has the largest impact on the constitutional system. It is not only laid down in the preamble and in article 2 where it appears as an abstract notion. It is also represented by the formula of "undivisibility of the State and its people" which can be found within the rules of limitation of the fundamental rights and even in the sections about the administrative organisation of the State. The Constitution of 1982 even gives answer to the question, how far the notion of nation is related to race or ethnos. The notion of a "State nation" seems to be in the foreground, when article 66 says that all those who are citizens of the Republic are Turks. This is exactly the notion of "Turkism" Mustafa Kemal Atatürk has fought for. On the other hand, the provisions on the of ficial language, the possibility to prohibite the use of other languages than Turkish (articles 26 and 28) and the foundation of the Atatürk High Society Institute of Culture, Language and History (article 134) which in practice constrains itself on the investigation of ethnic-Turkish language, history and culture, allow an ethnocentric (Turkish) interpretation of the principle of nationalism in the frame of the Turkish Constitution, as it is also reflected in the Turkish legislation. 
As a result based on Turkish constitutional and ordinary jurisprudence and doctrine, the constitutional principle of nationalism in Turkey does not allow a consequent interpretation. Both antiracist and ethnocentric elements can be discovered. In any case, it has to some extent to be understood as a serious limitation to the principles of a democratic society in a sense that has been defined under the jurisprudence of the organs of the European Convention on Human Rights and, of course, as a limitation to minority rights. 